\title{
Advanced microscopy techniques for a better understanding of the polymer/nanotube composite properties
}

\author{
K. Masenelli-Varlot, C. Gauthier, L. Chazeau, F. Dalmas, T. Epicier, J.Y. Cavaillé \\ INSA-Lyon, MATEIS CNRS UMR5510, F-69621 Villeurbanne, France
}

\subsection{Introduction}

During the last decades, polymer nanocomposites have become one of the most active research fields in polymer science. One of the driving forces of this research is the appearance of new nano-sized objects with interesting properties, like nano-clays, cellulose whiskers and carbon based nanofillers (fullerenes, vapor grown carbon fibers, carbon nanotubes) [1]. For instance, carbon nanotubes were first described in 1991 by the electron microscopist lijima who was studying the arcevaporation synthesis of fullerenes [2]. These long and thin cylinders of carbon can be seen as concentric graphene sheets (a hexagonal lattice of carbon) rolled into cylinders. Just a few nanometer across, these cylinders can be tens of $\mu \mathrm{m}$ long, and each end is "capped" with half of a fullerene molecule [3]. Nanotubes can have either a unique cylindrical wall (SWNTs) or multiple walls (MWNTs). Intrinsic mechanical properties of single-wall nanotubes have been theoretically predicted (Young's Modulus $1 \mathrm{TPa}[4,5]$, maximum tensile strength 30 GPa [6]). Moreover, they have excellent thermal conductivity, they are chemically inert and, depending on the details of their atomic arrangement (chirality), they behave as metals or semiconductors.

Carbon nanotubes have soon appeared as unavoidable candidates as reinforcing fillers in polymer composites. In addition to their intrinsic properties, they indeed display some of the specific features required for reinforcing fillers: one is their huge interfacial area (up to 100 to $1000 \mathrm{~m}^{2} / \mathrm{g}$ of fillers), that is known to favor the short distance filler-filler interaction, to increase the filler matrix interactions and even to modify molecular mobility of an important matrix fraction near the surface. Moreover, their high aspect ratio can lead to the formation of a percolating network at low nanotube content. In addition, the adhesion strength of the nanotube/polymer interface may also be a key factor for reinforcement, which explains that a lot of ongoing works have been focused on understanding the nature of nanotube/polymer matrix interactions and on the grafting of a polymer on nanotube surface $[7,8]$.

Undoubtedly, understanding the reinforcement of carbon nanotubes based nanocomposites requires the characterization of several parameters of the nanotubes like diameters, lengths (and 
their distribution), structures (SWNT, MWNT). Moreover, after processing, the three-dimensional distribution of nanotubes within the polymer matrix, the presence of agglomerates, the interfacial properties have also to be precisely characterized. However, CNT based nanocomposites are very difficult to characterize, because they must be observed at a very large scale range, from a few $\mathrm{nm}$ (CNT diameter) up to a few $\mu \mathrm{m}$ (CNT length). As a consequence, their characterization requires the combination of various techniques. The main techniques which intend to characterize the nanocomposite microstructures are, roughly speaking, divided into two groups, namely microscopy and scattering techniques. Scattering techniques lead to information through the use of modelling calculation of the interactions between nanoscopic heterogeneities and electromagnetic beams. The obtained information is an average over at least the whole volume in interaction with the beam (often in the order of $\mathrm{mm}^{2}$ ). In this chapter, we will rather focus on microscopy techniques, which present the advantage of providing direct and precise images.

In the following, we will review several imaging techniques, either based on beam/matter interactions (like electron microscopy either TEM or SEM) or techniques that probe the short distance interactions (like AFM), as they are well suited for the observation of nano-objects and have been extensively used to study nanotubes alone. The principles and the main results on polymer/nanotube composites provided by these imaging techniques (AFM, TEM and SEM) will be presented.

\subsection{Near Field Microscopies}

By comparison with electron microscopy, the main advantage of near field microscopy is to place a thin probe in the close vicinity of the sample, which avoids working in the propagation regime, and therefore avoids the limitation due to the use of an electromagnetic beam. This probe is located at a given distance from the sample in the nanometer range. To avoid any damage of the probe, a continuous control of the probe-to-sample distance is ensured. Data are processed from the detection of tip - sample interactions (distance, force, current, temperature, etc.) by scanning the sample surface (ensured by a piezoelectric system), a mapping of the chosen physical data can be obtained. Once the type of collected data is chosen, the lateral resolution mainly depends on the tip shape and its distance to the sample. Among the different local probes [9], two peculiar techniques have lateral resolution of $0.1 \mathrm{~nm}$ suited to the study of nanocomposites and will therefore be detailed: scanning tunneling microscope (STM) and atomic force microscopy (AFM).

\subsubsection{Principles of STM and AFM}

The first near-field microscope was a scanning tunneling microscope (STM), invented in 1982. It was based on the tunneling effect which is the occurrence of a current between two conducting or semi-conducting materials at a distance of few $\AA$ through an insulating medium, from the application of a voltage difference between them. The exponential dependence of this current with the tip-tosample distance leads to the very good resolution of this technique.

Different modes can be used. The continuous current mode consists in keeping the tunneling current constant through a control loop which continually adjusts the sample-to-probe distance. Spectroscopic measurement can also be performed during the sample surface scan. It consists, after adjusting the probe-sample distance, in applying a variable voltage to measure its influence on the tunneling current, which in turn provides information on the chemical characteristics of the sample. The STM technique can also be enriched by in situ measurements of the photon emission induced by 
the de-excitation of electrons ejected from the sample by the probe, enabling a fine analysis of the local optical properties.

Atomic force microscopy (AFM) was introduced a few years after as an application of scanning tunneling microscopy. By combining the principles of the STM with that of a profilometer, it enables imaging conductive or non-conductive sample surfaces with lateral and vertical resolutions of $30 \AA$ and $1 \AA$, respectively. The technique is based on the measurement of the interaction force between the tip and the sample surface. The force results from either the interaction potential between the sample and the tip atoms, or friction (during surface scanning). The force may also arise from magnetic or electrostatic interactions, if the tip is conducting or covered by a magnetic material.

The scheme of a typical AFM is presented in Figure 13.1. A cantilever holds the tip at one of its end. The tip position can be accurately measured from the reflection of a laser beam onto a set of four optical detectors. Basically, the relative displacement of the tip from the sample gives either access to the measured value, either to an isovalue surface when the loop controlled feedback adjusts the height of the sample surface to keep constant the value. In addition, the AFM tip can be static or oscillating (via its fixation on a piezoelectric device).

Different types of imaging can be obtained depending on whether the tip is in contact or not with the sample, on whether the cantilever is working at its resonance frequency or at zero frequency, and on whether the sample is vibrating or not. However, three main modes can be distinguished in AFM :

- Contact mode: in this mode, the cantilever is not vibrating. The tip is put in contact with the sample with a controlled load of the order of $10^{-8} \mathrm{~N}$. This mode is similar to that of a classical profilometer. Its drawback is the wear or the deformation of the sample surface it may generate.

- Resonating mode: in this mode the cantilever oscillates with small amplitude at its resonance frequency, "far" from the sample. The gradient of the surface interaction force shifts this resonance frequency towards higher values. Inversely, at a given excitation frequency, the oscillation amplitude is modified and gives information on the local force gradient. This mode is however rarely used for topographical study.

- A tapping mode (also called intermittent contact mode): it is a non linear resonance mode. In this case, the oscillation amplitude is larger and the mean position of the tip is closer to the surface. The tip almost touches the surface at each oscillation. In this mode, friction can be avoided as well as the sample deformation and wear. Adhesion is also avoided thanks to the extremely short time of "contact». The height of the sample is generally controlled so that the oscillation amplitude remains constant. The phase shift of the oscillation is then characteristic of the system dissipation, which is very useful for characterizing viscoelastic materials.

Other uses have been developed, as for instance the use of AFM tip as a local voltmeter. In that case, AFM becomes an Electrostatic Force Microscope (EFM). Measurements can be performed under the application of a voltage between the sample and the tip. In the dc-EFM mode, a constant voltage is applied to the sample; the AFM is operating in non contact mode with the cantilever oscillating near its resonant frequency. In the ac-EFM mode, the voltage is oscillating so that the cantilever always oscillates at its resonance frequency. Note that many different terminologies can be found to name apparatus coupling AFM principle with electrical/electrostatic properties of the imaged sample, depending on the control loop details and the apparatus provider.

Near field microscopy can also be combined with Raman spectroscopy to perform chemical analysis at the nanometer scale. Tip enhanced Raman Spectroscopy (TERS) combines the surfaceenhanced Raman scattering (SERS) and the high spatial resolution of AFM or STM. The metallic or 
metallized tip is illuminated by a focused laser beam and the resulting strongly enhanced electromagnetic field at the tip apex act as a highly confined light source for Raman spectroscopic measurements [10].

\subsubsection{Near field microscopy for nanotubes}

The properties of the nanotubes alone have been studied by AFM. Duesberg et al. measured the length distribution of nanotube samples and thus studied the influence of a purification process based on size exclusion chromatography [11]. AFM device can also be used as a nano-manipulator placed in a scanning electron microscope. Such nanomanipulation can be performed with high speed AFM coupled with a haptic device [12]. The first measurement of the stiffness of arc-MWNT pinned at one end was performed by Wong et al [13] who gave an average value of $1.28 \mathrm{TPa}$. They also conducted strength measurements, obtaining an average value of $14 \mathrm{GPa}$. The modulus was also deduced from bending measurement of an arc-MWNT pinned at each end over a hole, by Salvetat et al. [14]. The same type of measurement was also performed by Yu et al. still on MWNTs [15]. Experiments on SWNT were performed later by the same authors $[16,6]$.

STM has mainly been used to characterize the electronic properties of nanotubes [17]. For example, Bachtold et al used EFM in both ac and dc modes to probe the nature of conduction in SWNTs and MWNTs [18]. The electrical properties of SWNTs and MWNTs have been characterized by current sensing AFM (CS-AFM) by Dong et al. [19]. Rodriguez et al. [20] studied the same properties combining the CS-AFM with confocal Raman spectroscopy. New developments in TERS have been applied to carbon nanotubes by Bargioni et al. [21] and Roy and Williams [22]. For instance, TERS has been used to localize different single carbon nanotubes within a carbon nanotube bundle [23].

\subsubsection{AFM and CNT composites}

\section{Dispersion studies:}

AFM is a surface technique which needs flat surfaces, to avoid tip degradation during scanning. Such flat surfaces can be obtained from the sample surfaces directly after processing or from cryoultramicrotomy. The dispersion of MWNT in PE has thus been studied by AFM by McNally et al. [24]. In this example, the image was taken without any sample preparation. The obtained image evidenced the nanotubes alignment along the flow direction during melt compounding. AFM tapping image also enabled to check the dispersion of PS-grafted SWNT in PS [25] (see Figure 13.2). The contrast between the nanotubes and the polymer matrix can be enhanced by applying a voltage difference between the sample and the probe during a scan in tapping mode (dc-EFM) as shown in the study performed by Phang et al on MWNTs/PA6 composites [26]. The same authors also used the AFM in 'TUNA mode, that is as a STM but the obtained images are much less convincing. DC biased AFM was also used recently by Thompson et al. to image the dispersion of CNT in a poly(styrene bethylene butylene-b-styrene) matrix [27].

\section{Interfacial studies:}

Given the small diameter of the nanotubes, the evaluation of the interfacial shear strength between them and the polymer matrix is very difficult. Fortunately, interactions between nanotubes and polymer can be characterized by the use of the AFM as a nanomanipulator device. For instance, peel tests have been developed by attaching nanotubes on tips and putting them in contact with a polymer substrate. Strus et al [28] recently studied the peeling of MWNT from epoxy, graphite and polyimide substrate. Shear strength can also be deduced from nano pull-out tests. For instance, Barber et al. mounted an MWNT onto an AFM tip and then pushed it in a heated polymer film of 
poly(ethylene butene). After cooling, they measured the forces required to pull the tubes out, see Figure 13.3, and deduced stress values between 20 and $90 \mathrm{MPa}[29,30]$. They also studied the pullout behavior a MWNT from an epoxy matrix [31].

Despite the results presented above, near-field microscopy has not been extensively used to characterize polymer/nanotube composites However, it can be noticed that AFM is a useful technique to locally probe the mechanical properties of the composites (at the polymer-nanotube interface for example). One possible reason for the relatively small amount of studies by AFM and STM could be that observing the surface only (or the sub-surface) does not permit to obtain much information on the nanotube dispersion state. For that kind of characterization, transmission electron microscopy is a key technique owing to the small nanotube diameter.

\subsection{Transmission Electron Microscopy}

\subsubsection{Principles}

Transmission Electron Microscopy (TEM) is an imaging technique, where electrons pass through a very thin sample. The image and the electron gun are on opposite sides relative to the sample, as indicated by the keyword "Transmission". The main advantage of TEM relies in its resolving power. Indeed, resolution in a microscope is limited by diffraction phenomena. Thus, the image of a small point source will not be a point but a small bright spot, called the "Airy disc", surrounded by less intense bright and dark haloes. The resolving power is directly related to the size of the Airy disc, and thus on the incident beam wavelength. The wavelength associated to electrons in a TEM is so small that fine features can be distinguished, such as atomic columns [32].

The contrast in the image is formed from the interactions between the incident electrons and the matter. The most common operating mode in TEM is the bright field imaging mode. In this mode and in the case of non-crystalline materials such as polymers, the contrast in the image is the consequence of absorption of electrons in the sample and to a first approximation, the intensity at each position in the 2D projection of the sample may be modelled via Beer's law. Experimentally, thicker regions of the sample or regions with a higher atomic number will appear darker. For this reason, heterogeneous polymer samples are often observed after staining by heavy atoms, such as ruthenium or osmium, which selectively reacts with polymers depending on their chemical structure [33].

As far as crystals are concerned, the organized structure leads to diffraction of the incident electrons. In the low magnification images, a contrast arises from the selection of one spot only (in the diffraction plane), which will be used to form the image (see Figure 13.4). Depending on which spot is selected, the image is qualified as "bright-field" or "dark-field" image. In the bright field mode, the central spot, corresponding to no diffraction, is chosen and the regions where crystal planes can give rise to diffraction will appear in dark in the image. On the contrary in the dark field mode, a diffraction spot different from the direct beam is selected and only the regions where planes are in the corresponding Bragg position will appear in bright in the image [32]. Diffraction contrast can be used to identify lattice defects in crystals. Indeed, if the sample is oriented so that one particular plane is only slightly tilted away its Bragg angle, any distortion of the crystal plane that locally tilts the plane will produce particularly strong contrast variations.

Alternatively, images can be formed with electrons scattered at high angles (HAADF mode, for High Angle Annular Dark Field mode). An annular detector is placed below the sample and the 
focused electron beam is scanned over the sample. Interestingly, the contrast is not altered by diffraction phenomena and depends mainly on the atomic numbers of the atoms in the specimen [34].

At high magnification, TEM enables the formation of images displaying the atomic structure. It is thus qualified as "High Resolution TEM". The principle is that at least two diffraction spots are selected and the image is the interference pattern of the corresponding waves.

Whereas TEM images give insight on the materials structure, chemical information can be obtained via two coupled analysis techniques, namely Energy Dispersive Spectroscopy (EDS) and Electron Energy-Loss Spectroscopy (EELS). The EDS signal arises from X-ray photons emitted by the sample after inelastic interactions with the incident electrons. The heavier the atoms in the sample, the more intense the EDS signal. The X-ray photon energy directly depends on the sample composition. Thus, it is possible to determine the local composition (the spatial resolution being that of the microscope), and to acquire maps representing the spatial distribution of elements.

EELS is complementary to EDS since it is better suited to light elements. Indeed, the EELS signal consists in incident electrons collected onto the detector with different energies, since energy losses occur when electrons interact with atoms in the sample. The energy transferred from the incident electrons to the specimen serves to excite probed atoms, and the deposited quantity of energy depends on the chemical nature of atoms, but also on their electronic structure. Therefore, the EELS signal contains information not only on the sample composition but also on way atoms are bound to each other [35]. For conducting materials, the EELS signal is interpreted in terms of density of states. In the case of polymers, the use of chemical bondings is preferred [36]. When a 2D detector is used, images can be formed with electrons that underwent a specific energy loss (EFTEM mode, for Energy Filtered TEM). In that mode, maps can be calculated, representing the spatial distribution of elements [35] or even of chemical bondings [37]. EFTEM is also a very convenient way to gain contrast by suppressing the inelastic contribution of the signal in so-called 'zero-loss' or elastic images: this is of particular interest in thick samples and/or at low voltage since the inelastic signal increases and blurs the image.

In all modes, TEM provides two-dimensional images, corresponding to projections of the sample structure along the optical axis making ambiguous the interpretation of such images. On the contrary, TEM tomography (TEMT) generates 3D images with a nanometer scale resolution from tilt series of 2D projections acquired over a large angular amplitude, up to $120-130^{\circ}[38,39]$. Important advances in the microstructural analysis of heterogeneous polymers $[40,41]$ or polymeric nanocomposites $[42,43,44]$ have been recently achieved by TEMT. However, the resolution in the third direction in TEMT reconstructed volumes is very often limited by the "missing wedge" in tilt series acquisition. The precise quantification of the observed 3D microstructure is then strongly limited. Different strategies have been recently considered to solve this issue. From an experimental point of view, needle-like TEM samples have been, for instance, processed by Focused Ion Beam (FIB) technique, allowing a complete rotation of the sample and avoiding any missing angular observation [45]. On the other hand, several teams have developed new reconstruction algorithms in order to improve the reliability of the reconstruction $[46,47]$.

As long as amorphous materials are characterized, the TEM images can be easily acquired in the bright field mode, since the contrast in the image is mass-thickness dependent. However, for crystalline materials such as nanotubes, a contrast may appear due to diffraction, which may affect the quality of the volume reconstruction. Thus, the images may be acquired in the EFTEM or HAADF modes, if sufficient contrast from the nanotubes (mostly, depending upon their diameter) is obtained. 
The most recent TEM developments which may represent some interest for the study of nanotubes-based polymer nanocomposites are the optical corrector and the environmental mode. In a $\mathrm{C}_{\mathrm{s}}$-corrected imaging mode ( $\mathrm{C}_{\mathrm{s}}$ : spherical aberration coefficient), a better resolution is obtained, but also a sharper contrast which may improve the visibility of small objects. In an Environmental dedicated TEM, a differential pumping between the electron gun and the specimen stage allows the specimen to be surrounded by a gaseous atmosphere, with a pressure of up to a few millibars. Interestingly, the spatial resolution of the TEM is not seriously affected since atomic resolution is still attainable [48] and chemical reactions can be followed in situ. EELS can even be used to quantify the gas composition inside the reaction cell [49].

\subsubsection{Characterization of carbon nanotubes}

Historically, carbon nanotubes were first observed by Transmission Electron Microscopy, see Figure 13.5 [2]. Then, nanotubes have gained interest worldwide and led to an incredible number of published papers. It is simply not possible to give an exhaustive list of all studies involving TEM. However, a few ones can be highlighted because they focused on the characterization of the nanotube intrinsic properties, which in turn will play a great role on the polymer/composite properties.

\section{Nanotube structure:}

The first studies after lijima's paper, presenting TEM micrographs of carbon nanotubes (see Figure 13.5), mainly focused on the nanotube structure $[50,51,52,53]$ to determine how the graphene sheets roll themselves up. Once it has been established that the nanotubes were composed by concentric graphene sheets, one of the main issues, especially for single walled nanotubes, was the determination of the nanotube helicity $[54,55,56]$. At the same time, TEM studies were involved in the development of various elaboration processes $[57,58,59,60]$. The in situ growth of carbon nanotubes could even be followed in an environmental TEM, giving insights on the growth mechanisms $[61,62,63]$.

The structure of defects in nanotubes, which occur spontaneously or can be created by doping, has also been extensively studied $[64,65,66]$. In particular, several teams used EELS to probe the chemical bondings of nitrogen in nitrogen doped carbon nanotubes $[67,68]$.

\section{Quantification of the diameter distribution:}

One major issue of the nanotube elaboration processes is to produce nanotubes with controlled dimensions (diameter, length) and without byproducts (catalytic residues, other carbonaceous particles). This is nearly achieved with processes such as catalytic chemical vapor decomposition using supported catalysts. However, the arc discharge method remains the most common one and is perhaps the easiest way to produce the large quantities of nanotubes required for the elaboration of composites [69]. The dimensions of the nanotubes being uncontrolled, it is interesting to measure their distributions (for modelling purposes of the polymer/nanotube mechanical or electrical properties, for example). Unfortunately, the nanotube length cannot be accurately measured by TEM. They are indeed too long to be observed on their whole inside a single mesh of a microscopy grid. Moreover, they are generally so entangled that it is difficult to separate them from each other, especially in the presence of impurities or agglomerates. In a few cases, image analysis of TEM images can still be successfully performed and provide the diameter distribution as well as insights on the wall compacity [70]. 


\section{Nanotube properties:}

Whatever the nanotube production method, understanding the properties of materials filled with nanotubes requires the knowledge of the nanotube properties. As a consequence, many efforts were made to experimentally measure the nanotube Young modulus and the nanotube intrinsic conductivity. Fortunately in TEM, it was observed that the nanotubes were vibrating when clamped at one end and free at the other one (see Figure 13.6). Thus, the measurement of the mean-square vibration amplitude in function of the temperature allowed the determination of the Young modulus (higher than $1 \mathrm{TPa}$ for bundles of SWNTs) [71]. Alternatively, the vibrations can be electrically induced using specific specimen holders [72]. Other types of specimen holders also permitted to determine nanotube mechanical properties [73, 74, 75, 76].

As far as the nanotube conductivity is concerned, it obviously depends on the nanotube structure (MWNT or SWNT) and also on its helicity (in the case of SWNTs). TEM has also permitted to relate the helicity to the transport properties of nanotubes [77], the helicity being determined from electron diffraction patterns and the transport properties being probed with a dedicated TEM specimen holder.

\subsubsection{Characterization of polymer/nanotube composites}

Once the nanotubes are characterized and polymer/nanotubes elaborated, their microstructures have to be precisely determined to understand the relations between the process and the nanocomposites macroscopic properties. It is expected that the microstructural parameters that will play major roles (in addition to the filler geometry) are the nanotube dispersion and orientation state and the polymer/nanotube interfacial adhesion strength. In this section, we present a review of the pieces of information that can be brought by TEM.

\section{Nanotube dispersion state}

Obtaining a homogeneous nanotube dispersion state in a polymer matrix is still a major issue. From a general point of view, the dispersion state is influenced from the following parameters [78]:

- The nanotube length,

- The nanotube entanglement,

- The nanotube volume fraction,

- The matrix viscosity,

- The tube/tube attraction (mainly for single and double-walled nanotubes).

As a consequence, the nanotube dispersion state has to be characterized at several pertinent scales, including that covered by TEM. In particular, it can be noticed that in the case of a fine dispersion with very few agglomerates, it is hard to judge the grade of dispersion, because separated CNTs are more difficult to observe in bright-field TEM (especially for CNTs with few walls), as agglomerates giving better contrast. However, since alternative methods are not available to estimate the dispersion state, conclusions are always drawn from a few TEM images [78, 79, 80].

In some studies, a statistical description of the nanotube dispersion state was obtained from TEM images. For example, Uchida et al. [81] measured the diameter distribution of SWNT bundles in poly(acrylonitrile), with and without a purification treatment involving sonication in methanol. The different bundle diameter distributions (especially the mean diameter) could explain the different composite tensile moduli. Fornes et al. [82] also determined the diameter distribution of SWNTs bundles in a polymer matrix (namely polycarbonate). To improve the contrast in the bright-field TEM images and better measure the bundle diameter, they dissolved the polymer in chloroform and studied the remaining SWNT network. 
In order to understand the mechanical and electrical properties, the nanotube dispersion state has to be more precisely determined. Indeed, such properties depend on the formation of a threedimensional nanotube network. Once such a network is formed, key parameters include the nanotube curvature and the distance between entanglements. Electron tomography is undoubtedly the most pertinent technique to obtain a three-dimensional view of polymer/nanotube microstructures. For the moment, the published studies presenting electron tomography on polymer/nanotube composites have focused on how to obtain a good contrast between the nanotubes and the polymer matrix. Results have been presented either from bright-field [83, 84], HAADF [85] or from energy-filtered images $[86,87]$. The reconstructed volume presented in the study by M.H. Gass et al. [87] and reproduced in Figure 13.7 was obtained from a series of energyfiltered images at every tilt angle. The energy-loss spectrum was then determined at every image pixel. The difference in plasmon energy that exists between the two carbonaceous materials ( $22 \mathrm{eV}$ for nylon and $28 \mathrm{eV}$ for multiwalled carbon nanotubes) permitted to obtain a 'ratio image' that enhances the signal from the nanotubes. The volume was finally reconstructed from the "ratio" images at every tilt angle. This study emphasized that electron tomography is a powerful technique to study the three-dimensional structure of materials, even those exhibiting a poor contrast in bright field, such as polymer/nanotube composites.. Attempts to get a 3D view of the carbon nanotube network 3D structure were performed by Liu et al. [88], see Figure 13.8. In order to minimize any possible irradiation damage of the poly(styrene-co-butyl acrylate) P(S-BuA) latex matrix, tomography series were acquired at $80 \mathrm{kV}$. At such a low voltage in the TEM, energy-filtered imaging is almost compulsory in order to image correctly the CNTs at high tilt angles (owing to the severe thickness increase: $290 \%$ at $70^{\circ}$ ), as demonstrated by Figure 13.8). Although the thickness of the thin foil was relatively large $(200 \mathrm{~nm})$, the $3 \mathrm{D}$ reconstruction shows that it remains to small compared with the mean distance between the tubes, and especially the expected distance between two successive entanglements. It will be seen in section 13.4 that SEM tomography can be more appropriate for such a study.

From the point of view of the distribution of radius of curvature, nanotube networks were successfully investigated by Dalmas et al. [89]. Indeed, the 2D apparent nanotube segment curvature radius distribution was then measured on TEM bright field images acquired on the composites (see Figure 13.9). The experimental curvature population was then statistically compared to that measured on the projection of the simulated 3D microstructure (simulation of TEM observations). It was then used to model the nanocomposites electrical properties as a function of the elaboration process [90].

At this stage, it is worth mentioning that most of the previous studies were performed on homopolymers or random copolymers as matrices. Meincke et al. [91] observed that nanotubes were dispersed in the polyamide- 6 phase in blends of polyamide- 6 , acrylonitrile/butadiene/styrene (ABS). Unfortunately, no further quantification of the nanotube dispersion state has been presented, probably because of the non-spherical shape of the polyamide- 6 domains. Li et al. [92] used multiwalled carbon nanotubes as fillers in an extruded poly[styrene-b-(ethylene-co-butylene)-bstyrene] triblock copolymer (SBS). TEM observations confirmed small-angle X-ray scattering experiments: the well-dispersed MWCNTs have a marked effect on the phase separation behavior of the matrix block copolymer. They inhibit the local phase separation of SEBS, and the MWCNT network impedes the formation of large phase-separated grains. The relationships between the nanocomposites microstructures and properties were investigates. The role of the nanotube network on the mechanical and electrical properties was obvious. However, the change in the copolymer microstructure was not clearly evidenced. Similar nanocomposites were elaborated by Fragneaud et al. [93] but with polystyrene-grafted nitrogen-doped carbon multiwalled carbon nanotubes as fillers. It was also observed that the copolymer structure was altered by the nanotubes, the thinnest tubes being even incorporated into the copolymer polystyrene cylinders (see Figure 13.10). The higher 
electrical percolation threshold with a SBS matrix compared to that measured with a PS matrix can most probably be attributed to the copolymer structure.

\section{Nanotube orientation state}

Although nanotubes tend to align themselves as soon as shear is applied during the nanocomposites elaboration process, very few studies focus on the quantification of the nanotube orientation state. The method proposed by Zhihang et al. [94] is worth mentioning because it can easily be used and the graphical representation allows a rapid understanding. Basically, the orientation of a nanotube can be characterized by a unit vector (collinear to the nanotube), see Figure 13.11. Experimentally, the angles between the unit vectors and a reference direction are measured on TEM images and an ellipse is drawn to graphically gather the orientations of all the unit vectors. In the ellipse, the major axis represents the preferred orientation of nanotubes in the analyzed region. The ratio between the major and minor axes distances of the ellipse represents the degree of orientation in that direction. Thus an elongated ellipse indicates a high degree of alignment in the direction of the major axis, whereas a circle signifies an orthotropic orientation.

\section{Polymer/nanotube interfacial adhesion strength}

The interfacial adhesion strength is undoubtedly the most difficult to characterize and over all to quantify. A first method consists in observing the composite behavior during the preparation of a TEM sample. Thin sections of polymer-based materials are generally prepared by ultramicrotomy, that is by direct cutting with a diamond knife. We observed that raw MWNTs were hardly cut and were instead pulled out from the polystyrene matrix, resulting in holes during observation (see Figure 13.12a). On the contrary, polystyrene-grafted nitrogen-doped multiwalled nanotubes fragments were easily observed in the TEM sample (see Figure 13.12b), which clearly indicates an improvement in the interfacial adhesion strength. The raw nitrogen-doped multiwalled nanotubes were found to be an intermediate case, with observable fragments but still a lot of holes in the sample [95].

A second method consists in observing the composite fracture surfaces. For example, in the case of MWNTs in a poly(hydroxyaminoether) matrix, C. Bower et al. [96] observed a lot of pulled out nanotubes and concluded that the load transfer from polymer to nanotube was not sufficient to fracture the nanotubes. In the same time, a lot of kinked MWNTs were observed, which were believed to be plastically deformed.

Information can also be indirectly obtained by TEM when observing a thin section of the composite. Indeed, damage occurs during observation because of electron-matter interactions, which generally results in strains in the sample. Under certain conditions, deformation of the sample under electron irradiation can be assimilated to local tensile tests. Composites made of polystyrene and MWNTs were studied by D. Quian et al. [97]. They followed the composite behavior after crack initiation due to electron irradiation and estimated that about half of the aligned nanotubes have been broken. In agreement with theoretical calculations of the composite modulus (randomly oriented discontinuous fiber model), it was concluded that with polystyrene, load could be successfully transferred to the nanotubes through the nanotube-polystyrene interface. However, it can be pointed out that the remaining half of the nanotubes were still pulled out from the matrix, which indicates that the load transfer and thus the interfacial adhesion are not the highest possible. As far as SWNTs are concerned, Lourie et al. [98] also concluded that there was a significant interfacial adhesion with epoxy, since the SWNTs were broken instead of pulled out. The absence of pull-out mechanism was attributed to a higher reactivity of the graphene sheets when highly curved into SWNTs. 
The poor quality of the interfacial adhesion between polymers and MWNTs was one reason of the development of functionalization and grafting. The aim is to make chemical bondings (on functionalized nanotubes) or layers of grafted polymer (on grafted nanotubes) appear, that will improve the interfacial adhesion. Two studies involving "tensile tests" under electron irradiation are worth mentioning. In the first one, by Gojny et al. [99], the deformation mechanisms of composites with epoxy and raw or oxidized MWNTs were compared. As the raw nanotubes were pulled out from the epoxy matrix, telescopic pull-outs were observed with functionalized nanotubes. This clearly indicates that the interfacial adhesion was significantly improved. A similar conclusion was drawn by Hwang et al. [100] when studying poly(methyl metacrylate) (PMMA) filled with PMMA-grafted MWNTs.

At this stage, it is worth mentioning that functionalization or grafting can also affect the nanotube dispersion state. From a macroscopic point of view, the mechanical and electrical properties are also expected to be modified. A full understanding of these macroscopic properties implies to characterize the nanotube dispersion state, the polymer-nanotube interfacial adhesion strength, more precisely than what has been done in the literature until now. In particular, quantitative data should be deduced from the images, such as the distance between entanglements for example. Moreover, as far as grafted nanotubes are concerned, an evidence of grafting often lacks and it has to be proved that the polymer has been successfully grafted onto the nanotube, instead of a nanotube wrapping. TEM coupled with EELS and EDS is probably the best suited technique to locally investigate the grafted layer. For example, in Fragneaud et al. work [8], we probed the layer grafted onto nitrogen-doped nanotubes and proved by EELS that it was composed by polystyrene, as expected. Moreover, a peak assigned to bromine was detected on the EDS spectra, indicative of the successful atom-transfer radical polymerization. Further work is needed but since chemical bondings can also be determined from the EELS spectra, one can also expect quantifying the number of grafted chains.

As was detailed in this section, TEM can bring numerous pieces of information regarding the polymer/nanotube composite microstructure. However, it has to be recalled that nanofillers such as nanotubes easily agglomerates and their dispersion state has to be characterized from the micron to the nanometer scale. This is one reason, among others, why Scanning Electron Microscopy is another widely used to characterize polymer/nanotube composites.

\subsection{Scanning Electron Microscopy}

\subsubsection{Overview of the technique (SEI, BEI, CCI)}

Scanning Electron Microscopy (SEM) is a powerful tool for the surface observation of samples. The scanning of a small electron probe on the surface of the sample induces the emission of several signals. The main imaging mode used in SEM is based on the detection of the secondary electrons (called SEI for Secondary Electrons Imaging), giving an idea of the sample topography. Moreover, two other signals resulting from the primary electrons/sample interaction are also often used: backscattered electrons and X-rays, providing information about the topography and/or the composition of the sample (see Figure 13.13). 
As SEMs are versatile instruments for relatively low costs, they are largely available in laboratories in the field of biology and materials science. Developed in the 1940's, it has undergo many changes regarding the ease of use, the electron guns and optics, the resolution improvement and the development of specific modes such as transmission (STEM), low-voltage, environmental or variable-pressure, etc.

\subsubsection{Application to the study of nanotubes}

The resolution of SEMs is now suitable for nano-materials characterization. High resolution SEM is a powerful instrument for imaging of fine structures of materials and nanoparticles fabricated by nanotechnology. In lens SE, BSE modes, and STEM mode are often performed to check the structure of CNT growths or CNT as delivered by commercial producers, and sometimes coupled with TEM. Even the single-walled carbon nanotubes can easily be observed by HR-SEM (see Figure 13.14). The STEM mode can also be used for free CNT observation [101]. As in TEM, specific specimen holders have been developed to perform in situ mechanical tests. For example, Maschmann et al. carried out nanoindentation tests on nanotube arrays [102]. They showed that $7.5 \mu \mathrm{m}$ carbon nanotube arrays could significantly recover even after $90 \%$ compressive strain, whereas $600 \mu \mathrm{m}$ carbon nanotube arrays underwent significant plastic deformation at $15 \%$ strain.

The SEM contrast of CNT can be difficult to interpret as it depends on a lot of parameters [103]: the primary beam energy landing, the history of imaging, if the CNT are lying on a substrate or suspended, the substrate electrical conductivity and the electron beam induced contamination during imaging. The contrast can for example be linked to potential differences between the CNT and the substrate [104], or to electron beam-induced current on the insulating substrate surface [105].

\subsubsection{For polymer CNT/nanocomposites}

CNT nanocomposites morphological and structural analysis is often done by TEM but an extensive imaging is required then to ensure a representative view of the material. Moreover, carbon based fillers have very low TEM contrast when embedded in a polymer matrix. The application of microscopy techniques is very useful to control the status of CNTs at any time during the preparation process of $\mathrm{CNT} /$ polymer nanocomposites, and moreover, to gain insights on parameters important for a better understanding the performance of the final nanocomposite material based on CNTs.

The general approach in SEM is the direct observation of the surface of nanocomposites films or of fracture surfaces performed at ambient temperature or in liquid nitrogen, to check concentration, alignment and orientation distribution of the fillers, and to have information about the interface strength between the fillers and the matrix $[106,107,93,108]$, see Figure 13.15. However, SEM of carbon nanotubes embedded in a polymer insulating matrix can be a challenge: traditional gold-coating performed to prevent charging can sometimes mask the nanotubes and the alternatives include lower energies or environmental mode but may restrict the resolution [109].

SEM, as others surface-based methods such as scanning probe microscopy (SPM), or more specifically atomic force microscopy (AFM), generally only shows the surface or a cross-section of the three-dimensional arrangement of the CNTs in the polymer matrix. However, the team of Loos et al. has shown that conventional SEM is capable to provide (pseudo) three-dimensional morphological information on SWNT networks in conductive SWNT/PS nanocomposites at nanometric resolution by monitoring the sample in the charge contrast imaging mode (CCI) [110]. This is only observed for nanocomposites above the percolation threshold. With increasing acceleration voltage, the 
secondary electron yield at the positions of the SWNTs increases and enhances the contrast between SWNTs and the matrix, see Figure 13.16.

Reimer et al. have characterized specific samples in various transmission modes and have highlighted a beam broadening through the sample thickness. They have shown that the resolution, directly related to the probe size, is subjected to a top-bottom effect [111]. The use of a thin sample thus results in an improved resolution, avoiding the probe broadening through the sample thickness. Moreover, the interaction cross-sections are important at low voltage, for instance the cross section of carbon at $30 \mathrm{kV}$ equals the one of $\mathrm{Zn}$ at $100 \mathrm{kV}$, and the cross section of carbon at $10 \mathrm{kV}$ equals the one of Ta at $100 \mathrm{kV}$ [112]. These high values coupled with the high collection angles of our detection configuration can be interpreted as efficiency in both the electrons-sample interaction and the scattered electrons detection. This enables for instance polymer/carbon nanotube nanocomposites to be observed with an important contrast between the polymer matrix and the fillers, even if their average atomic numbers are very close (see Figure 13.17). In the STEM configuration, the detection efficiency also contributes to increase the thickness of transparency which can reach several $\mu \mathrm{m}$ [113].

The possibility to observe in SEM "thick" samples through STEM observations led to the development of different sample stages, which have been successfully used for the characterization of CNT and CNT polymer nanocomposites.

An imaging mode called "wet-STEM" developed in ESEM, and schematically presented in Figure 13.18, allows the observation of nano-objects suspended in a liquid phase, with a few nanometers resolution $[113,114]$. ESEM is indeed interesting for the ability to keep samples wet or liquid, and the STEM mode results in high contrast, resolution and thickness of transparency. At the steps before the elaboration of carbon nanotube nanocomposites, wet-STEM can be used for the characterization of nanotubes dispersed in a liquid (see Figure 13.19), and for polymer latex/nanotubes mixing (before evaporation or freeze-drying to elaborate polymer/carbon nanotube nanocomposites).

A rotating sample stage has also been developed to performed electron tomography in the ESEM, see Figure $13.20[115,116]$. Its main interest lies in the good compromise between the resolution level of a few $\mathrm{nm}$, and the large tomogram size thanks to the important thickness of transparency. Electron tomography in the ESEM is well adapted for non-conductive samples, and exhibits good contrast even for low-atomic number materials. Taking advantage of the size of the ESEM chamber, the range of tilt angles is not limited by the space around the sample. It has been used for the characterization of free nanowires, and for impact modified polymer composites [116] and more recently to wet samples [117]. Owing to the good contrast in the SEM-STEM mode even for rather thick specimens, the CNT-CNT contacts in polymer nanocomposites have been characterized and quantified by this technique. Tilted tomography was then performed on $0.2,0.5$ and event $1 \mu \mathrm{m}$ thick ultramicrotomic section of P(S-BuA)-CNTs latex-based nanocomposites. In complement to the results obtained with an ESEM, which offers the great advantage of compensating charges at the surface without any conducting coating, slightly better 'STEM' micrographs, in terms of resolution, and despite a few charging effects, were also acquired using a dedicated High Resolution SEM instrument (see Figure 13.21) [88]. These tomograms were analyzed with the help of a home-made software in order to quantify the surface fraction of contacts between tubes in order to establish a correlation between different conditions of elaboration of two-types of nanocomposites and their electrical conductivity (Figure 13.22). At this stage, the worse resolution of SEM tomograms compared to TEM tomograms (see Figure 3.X) can be considered as an advantage because it helped the numeric treatment of the images for their binarisation and the modelling of tubes as wavy cylinders [118]. 


\subsection{Focused Ion Beam Microscopy}

The FIB-SEM approach takes advantage of the recent developments in SEM, providing double gun microscopes. In such instruments, a focused ion beam (FIB) can be used to create a cut at a designated site in the specimen, followed by viewing the newly generated surface with a scanning electron beam focused at the same point [119 and recent review in 120]. The repetition of such a 'slice-and-view' approach in a sequential way permits a 3D FIB-SEM tomographic analysis of materials. This opens up the third dimension by making a SEM combined with a FIB a well suited tool for characterizing micron and sub-micron size microstructural features in three dimensions via serialsectioning procedures [121]. This approach can help to better characterize the dispersion and orientation of fillers in polymer nanocomposites. An example of such studies is illustrated in Figure 13.23a) [122]. The material consists of a polyurethane matrix reinforced by $\mathrm{Fe}_{3} \mathrm{C} @ \mathrm{C}$ nanowires; its microstructure resembles that of a CNTs nanocomposite. In this case, a good contrast is obtained between the fillers and the matrix in most of SEM imaging modes. The volume reconstructed from the 3D FIB-SEM sequence is seen at some angle to point out the trend to alignment of most fillers, which cannot be easily identified in conventional 2D imaging. Another example concerns a PET matrix filled with nanoclays: compared to conventional TEM techniques, a wider area of the sample can be observed when using FIB milling and imaging of the milled surface [123].

The main drawbacks of the FIB-SEM tomography are the low availability of such instruments in laboratories, and the fact that many samples require specific preparation [124], and can exhibit poor contrast in the case of polymers materials reinforced by fillers with low atomic density, as it is the case of carbon nanotubes. Most stringent is the generally poor resistance of these materials to the ion beam damage. These limitations are illustrated in Figure 13.23b): attempts to produce reasonable image stacks from $\mathrm{P}(\mathrm{S}-\mathrm{BuA})$-CNTs nanocomposites studied previously by TEM and SEM (see sections 13.3.3 and 13.4.3) were so far unsuccessful. At first, the matrix rapidly deforms under the ion beam, whatever the slicing conditions; secondly, because of the low contrast of the nanotubes, which is very similar to the so-called 'curtain effects' [125] produced by the ion beam: roughly vertical bands appear during milling, owing to the masking effect due to metallic catalysts present in the nanotubes population. The lines have a low contrast but similar to that of the nanotubes, making any image segmentation practically impossible. All these reasons probably explain why, even if the FIB/SEM approach is used on polymer nanocomposites, it is not used in the literature for carbon nanotubes in polymer matrix.

\subsection{Conclusions}

The main goal in material science is to provide behavior laws, i.e. to be able to predict the material properties under given conditions (mechanical, electrical, environmental conditions, temperature, etc.). This requires relating microscopic parameters and local mechanisms to macroscopic behaviors, as there is no other way to express such behavior laws based on chemicalphysical parameters. In other words, the study of materials requires a large part of microstructural observation and analysis.

More specifically with nanocomposite materials, and as recalled above, in most of the cases, interface between as received particles and organic matrices are very weak (bad adhesion), and one way to improve it consists in performing chemical grafting on the particle surface. If the chemical 
reactions are generally classical and work in solution, they can be much more difficult to achieve in heterogeneous media, which is the case for particle grafting. It is thus challenging to check the grafting effectiveness, and microscopies combining spectroscopy is probably the most powerful tool for such purpose.

We have roughly described three main microscopy techniques, namely local probe microscopy (STM, AFM, etc.), scanning electron microscopy (SEM) and transmission microscopy (TEM).

It has been pointed out that most of the time, microscopies lead to 2D images, either because they provide surface characterization (local probe and SEM), or because images result from the ability of electrons to cross the sample (TEM). Recent developments allow getting 3D images, by using for instance the classical tomography technique previously developed for $\mathrm{X}$-ray medical applications. It is worthy to note that an old and very tough technique firstly developed to observe in 3D bio-objects -such as viruses- has been recently renewed. In FIBs, an ion beam replaces microtome in producing thin slices, and in situ observation by SEM avoids manually changing the samples.

Scanning Probe microscopies are extremely useful for analyzing surfaces, but cannot lead to bulk information. They will be used each time surface properties are important, i.e. when surfaces are used for themselves (tribological applications, adhesion, etc.). However, in some cases, the study of transport phenomena (such as thermal or electrical conductivity) by modified AFM may lead to bulk characterization such as the formation of a percolating nanotube network for instance.

A similar conclusion could be reached for scanning electron microscopy, but two specific features must be underlined. The fact that CNT are good electrical conductors makes easier observations of CNT nanocomposites, even at low CNT volume fractions, provided they form a percolating network. In such cases, it appears that SEM observations show not only the nanocomposite surface topology, but also the CNT arrangement near the surface within a thickness of even few $\mu \mathrm{m}$. For too low volume fractions (dilute state of CNT), the sample surface must be coated, which removes all interest of their observation. On the other hand, as for other electron microscopies, spectroscopy analysis can be used for imaging purposes.

TEM remains certainly the most powerful technique to get bulk information, but due to the low sample thickness required for observation, in most cases, CNT are cut and it is almost impossible to observe them surrounded by their neighbors and so, to analyze their mutual interactions. However, in situ spectroscopy leads to more and more precise data on CNT - matrix interface, which one of the key-point of macroscopic behavior. It can be noticed that SEM can be performed in transmission, leading to images similar to what can be obtained by TEM. However, in the magnification range covered by both techniques, SEM provides images of thicker samples with a higher contrast, which should provide reliable results on the nanotube dispersion state. However, TEM remains unavoidable to locally characterize the nanotube-matrix interface and the nanotubenanotube contacts.

To summarize recent improvements which in fact appear as breakthrough, two limits of electron microscopy techniques have been recently bypassed, (i) one related to SEM observation of insulating materials and liquid states by using environmental microscopes, and (ii) the second one related to $3 \mathrm{D}$ rather than $2 \mathrm{D}$ imaging, by developing tomography techniques in microscopes working in transmission modes or by using micromachining in dual beam microscopes (FIB). Even if it remains delicate to apply this technique to beam-sensitive materials, we can expect that within few years, with the help of this new approach and many others, it will be possible to answer several questions, as for instance, the effect of nanotubes dispersion on nanocomposite mechanical properties or on electrical properties. 


\subsection{Acknowledgements}

This chapter is dedicated to Dr. A. Bogner-Van de Moortèle ${ }^{\dagger}$ who intensively worked on the first edition. Thanks are due to the CLYM for access to different electron microscopes used by coworkers of the MATEIS laboratory. The authors thanks Y. Liu (MATEIS) and K. Sato and T. Konno from the IMR, Tohoku University (Sendai-Japan) for their collaboration in the EFTEM and HR-SEM tomography works reported in sections 3.2 and 3.3.

\subsection{References}

1 L. Chazeau, C. Gauthier, G. Vigier, J.-Y Cavaillé, "Relationships between microstructural aspects and mechanical properties in polymer based nanocomposites", in "Handbook of organic-inorganic hybrid materials and nanocomposites, Pt2", Dr. H.S. Nalwa ed., American Scientific Publishers, 2003.

2 S. lijima, Nature, vol. 354, p. 56-58, 1991.

3 T. Ebbesen, "Carbon nanotubes: Preparation and properties", CRC Press, Boca Raton, Florida, 1997.

4 E. Dujardin, T. W. Ebbesen, A. Krishnan, P. N. Yianilos, M. M. J. Treacy, Phys. Rev. B, vol. 58, p. 1401??, 1998.

5 E.T. Thostenson, Z. Ren, T.W. Chou, Compos. Sci. Technol., vol. 61, p. 1899, 2001.

6 .M. F. Yu, B. S. Files, S. Arepalli, R. S. Ruoff, Phys. Rev. Lett., vol. 84, p. 5552, 2000.

7 M. Dehonor Gomez, K. Masenelli-Varlot, A. Gonzalez-Montiel, C. Gauthier, J.Y. Cavaillé, H. Terrones, M. Terrones, Chem. Commun., p. 5349-5351, 2005.

8 B. Fragneaud, K. Masenelli-Varlot, A. Gonzalez-Montiel, M. Terrones, J.Y. Cavaillé, Chem. Phys. Lett., vol. 419, p. 567-573, 2006

9 A. Yacoot, L. Koenders, J. Phys. D, vol. 41, p. 103001, 2008.

10 T. Schmidt, L. Opilik, C. Blum, R. Zenobi, Angewandte Reviews, vol. 57, p. 5940, 2013.

11 G.S. Duesberg, J. Muster, V. Krstic, M. Burghard, S. Roth, Appl. Phys. A, vol. 67, p. 117-119, 1998.

12 F. Iwata, Y. Oshadi, I. Ishikasi, L.M. Pico, T. Ushiki, Ultramicroscopy, vol. 133, p. 88, 2013.

13 E.W. Wong, P.E. Sheehan, C.M. Lieber, Science, vol. 277, p. 1971, 1997.

14 J.P. Salvetat, A.J. Kulik, J.M. Bonard, G.A.D. Briggs, T. Stockli, K. Metenier, S. Bonnamy, F. Beguin, N.A. Burnham, L. Forro, Adv. Mater., vol. 11, p. 161, 1999.

15 M.F. Yu, O. Lourie, M.J. Dyer, K. Moloni, T.F. Kelly, R.S. Ruoff, Science, vol. 287, p. 637, 2000.

16 J.P. Salvetat, G.A.D. Briggs, J.M. Bonard, R.R. Bacsa, A.J. Kulik, T. Stockli, N.A. Burnham, L. Forro, Phys. Rev. Lett., vol. 82, p. 944, 1999.

17 L.C. Venema, J.W.G. Wildoer, C. Dekker, G.A. Rinzler, R.E. Smalley, Appl. Phys. A-Mater. Sci. Process., Vol. 66, p. S153, 1998.

18 A. Bachtold, M.S. Fuhrer, S. Plyasunov, M. Forero, E.H. Anderson, A. Zettl, P.L. McEuen, Phys. Rev. Lett., vol. 84, p. 6082, 2000.

19 Z. Dong, U.C. Wejinya, S.N.S. Chalamalasetty, Sensors and Actuators A: Physical, vol. 173, p. 293, 2012.

20 R.D. Rodriguez, M. Toader, S. Hermann, E. Sheremet, S. Muller, O.D.Gordan, H. Yu, S.E. Schultz, M. Hietschold, D. R.T.Zahn, Nanoscale Research Letter, vol. 7, p. 682, 2012.

21 A. Weber-Bargioni, A. Schwartzberg, M. Cornaglia, A. Ismach, J.J. Urban, Y. Pang, R. Gordon, J. Bokor, M.B. Salmeron, D.F. Ogletree, S. Cabrini, P.J. Schuck, Nano Letters, vol. 11, p. 1201, 2011.

22 D. Roy, C. Williams, J. Vacuum Science Technology A, vol. 28, p. 472, 2010. 
23 N. Peica, C. Thomsen, J. Maultszch, Nanoscale Research Letter, vol. 6, p. 1269, 2006.

24 T. McNally, P. Potschke, P. Halley, M. Murphy, D. Martin, S.E.J. Bell, G.P. Brennan, D. Bein, P. Lemoine, J.P. Quinn, Polymer, vol. 46, p. 8222, 2005.

25 G. Viswanathan, N. Chakrapani, H.C. Yang, B.Q. Wei, H.S. Chung, K.W. Cho, C.Y. Ryu, P.M. Ajayan, J. Am. Chem. Soc., vol. 125, p. 9258, 2003.

26 I.Y. Phang, T.X. Liu, W.D. Zhang, H. Schonherr, G.J. Vancso, Eur. Polym. J., vol. 43, p. 4136, 2007.

27 H.T. Thompson, F. Barroso Bujans, J. Gomez Herrero, R. Reifenberger, A. Raman, Nanotechnology, vol. 24, p. 135701, 2013.

28 M.C. Strus, C.I. Cano, R.B. Pipes, C.V. Nguyen, A. Raman, Compos. Sci. Technol., vol. 69, p. 1580, 2009.

29 A.H. Barber, S.R. Cohen, S. Kenig, H.D. Wagner, Compos. Sci. Technol., vol. 64, p. 2283, 2004.

30 A.H. Barber, S.R. Cohen, H.D. Wagner, Appl. Phys. Lett., vol. 82, p. 4140, 2003.

31 A.H. Barber, S.R. Cohen, A. Eitan, L.S. Schadler, H.D. Wagner, Adv. Mater., vol. 18, p.83, 2006.

32 L. Reimer, H. Kohl, "Transmission Electron Microscopy: physics of image formation" $5^{\text {th }}$ edition, Springer Series in Optical Science, vol. 36, Springer, 2008.

33 L.C. Sawyer, D.T. Grubb, "Polymer Microscopy" $2^{\text {nd }}$ edition, Chapman \& Hall, 1996.

34 S. Pennycook, Ultramicroscopy, vol. 30, p. 58-69, 1989.

35 R.F. Egerton, "Electron Energy-Loss Spectroscopy in the Electron Microscope" $2^{\text {nd }}$ edition, Kluwer Academic/plenum publishers, 1996.

36 K. Varlot, J.M. Martin, D. Gonbeau, C. Quet, Polymer, vol. 40, p. 5691-5697, 1999.

37 J.M. Martin, B. Vacher, L. Ponsonnet, V. Dupuis, Ultramicroscopy, vol. 65, p. 229-238, 1996.

38 P.A. Midgley, M. Weyland, Ultramicroscopy, vol. 96, p. 413-431, 2003.

39 P.A. Midgley, E.P.W. Ward, A.B. Hungria, J.M. Thomas, Chemical Society Reviews, vol. 36, p. 14771494, 2007.

40 S. Akasaka, T. Okamoto, T. Osaka, T. Matsushita, H. Hasegawa, Eur. Polym. J., vol. 47, p. 651-661, 2010.

41 F. Dalmas, E. Leroy, Macromolecules, vol. 44, p. 8093-8099, 2011.

42 J. Loos, E. Sourty, K. Lu, B. Freitag, D. Tang, D. Wall, Nano Lett., vol. 9, p. 1704-1708, 2009.

43 K. Lu, E. Sourty, R. Guerra, G. Bar, J. Loos, Macromolecules, vol. 43, p. 1444-1448, 2010.

44 L. F. Drummy, Y. C. Wang, R. Schoenmakers, K. May, M. Jackson, H. Koerner, B. L. Farmer, B. Mauryama, R. A. Vaia, Macromolecules, vol. 41, p. 2135-2143, 2008.

45 M. Kato, N. Kawase, T. Kaneko, S. Toh, S. Matsumura, H. Jinnai, Ultramicroscopy, vol. 108, p. 221229, 2008.

46 B. Goris, T. Roelandts, K. J. Batenburg, H. Heidari Mezerji, S. Bals, Ultramicroscopy, vol. 127, p. 4047, 2013.

47 J.J. Fernandez, Current Opinion in Solid State and Materials Science, vol. 17, p. 93-106, 2013.

48 http://www.hitachi-hhta.com/sites/default/files/technotes/E-TEM-MicroscopyToday-XF06(2).pdf

49 P.A. Crozier, S. Chenna, Ultramicroscopy, vol. 111, p. 177-185, 2011.

50 X.D. Fan, L.A. Bursill, Phil. Mag. A, vol. 72, p. 139-159, 1995.

51 S. Xie, N. Li, Z. Zhang, W. Liu, G. Wang, S. Qian, C. Fu, J. Mater. Sci., vol. 30, p. 2291-2295, 1995.

52 S. Wang, D. Zhou, Chem. Phys. Lett., vol. 225, p. 165-169, 1994.

53 D. Ugarte, Microscopy Microanalysis Microstructures, vol. 4, p. 505-512, 1993.

54 M. Liu, J.M. Cowley, Carbon, vol. 32, p. 393-403, 1994. 
55 L.C. Qin, S. lijima, H. Kataura, Y. Maniwa, S. Suzuki, Y. Achiba, Chem. Phys. Lett., vol. 268, p. 101106, 1997.

56 L. Henrard, A. Loiseau, C. Journet, P. Bernier, Synthetic Metals, vol. 103, p. 2533-2536, 1999.

57 A. Loiseau, J. Gavillet, F. Ducastelle, J. Thibault, O. Stéphan, P. Bernier, S. Thair, C.R. Physique, vol. 4, p. 975-991, 2003.

58 P. Chen, H.B. Zhang, G.D. Lin, Q. Hong and K.R. Tsai, Carbon, vol. 35, p. 1495-1501, 1997.

59 P.M. Ajayan, J.M. Lambert, P. Bernier, L. Barbedette, C. Colliex, J.M. Planeix, Chem. Phys. Lett., vol. 215, p. 509-517, 1993.

60 K. Hernadi, A. Fonseca, P. Piedigrosso, M. Delvauw, J.B. Nagy, D. Bernaerts, J. Riga, Catalysis Letters, vol. 48, p. 229-238, 1997.

61 S. Helveg, C. López-Cartes, J. Sehested, P.L. Hansen, B.S. Clausen, J.R. Rostrup-Nielsen, F. AbildPedersen, J.K. Nørskov, Nature, vol. 427, p. 426-429, 2004.

62 R. Sharma, Z. Iqbal, Appl. Phys. Lett., vol. 84, p. 990-992, 2004.

63 S. Hoffmann, R. Sharma, C. Ducati, G. Du, C. Mattevi, C. Cepek, M. Cantoro, S. Pisana, A. Parvez, F. Cervantes-Sodi, A.C. Ferrari, R. Dunin-Borkowski, S. Lizzit, L. Petaccia, A. Goldoni, J. Robertson, Nanoletters, vol. 7, p. 602-608, 2007.

64 S. Amelinckx, A. Lucas, P. Lambin, Rep. Prog. Phys. Vol ? 62, p. 1471-1524, 1999.

65 D. Cherns, W.T. Young, F.A. Ponce, Materials Science and Engineering B, Solid-state materials for advanced technology, vol. 50, n 1-3, p. 76-81, 1997.

66 O. Stephan, P.M. Ajayan, C. Colliex, P. Redlich, J.M. Lambert, P. Bernier, P. Lefin, Science, vol. 266, p. 1683-1685, 1994.

67 R. Czerw, M. Terrones, J.C. Charlier, X. Blase, B. Foley, R. Kamalakaran, N. Grobert, H. Terrones, D. Tekleab, P. M. Ajayan, W. Blau, M. Ruehle and D. L. Carroll, Nano Lett., vol. 1, p. 457-260, 2001.

68 M. Glerup, M. Castignolles, M. Holzinger, G. Hug, A. Loiseau, P. Bernier, Chem. Commun., p. 25422543, 2003.

69 A. Loiseau, X. Blase, J.C. Charlier, P. Gadelle, C. Journet, C. Laurent, A. Peigney, "Synthesis methods and growth mechanisms", in "Understanding Carbon nanotubes. From basics to application ", Lecture Notes in Physics 677, Springer, 2006.

70 C. Gommes, S. Blacher, K. Masenelli-Varlot, C. Bossuot, E. McRae, A. Fonseca, J.B. Nagy, J.P. Pirard, Carbon, vol. 41, p. 2561-2572, 2003.

71 M.M. Treacy, T.W. Ebbesen, J.M. Gibson, Nature, vol. 381, p. 678-680, 1996.

72 P. Poncharal, Z.L. Wang, D. Ugarte, W.A. de Heer, Science, vol. 283, p. 1513-1516, 1999.

73 B.G. Demczyk, Y.M. Wang, J. Cumings, M. Hetman, W. Han, A. Zettl, R.O. Ritchie, Mater. Sci. Engin. A, vol. 334, p. 173-178, 2002.

74 Y. Zhu, H.D. Espinosa, PNAS, vol. 102, p. 14503-14508, 2005.

75 D. Golberg, X.D. Bai, M. Mitome, C.C. Tang, C.Y. Zhi, Y. Bando, Acta. Mater., vol. 55, p. 1293-1298, 2007.

76 P. Jaroenapibal, KKU Engineering Journal, vol. 40, p. 131-138, 2013.

77 M. Kociak, K. Suenaga, K. Hirahara, Y. Saito, T. Nakahira, S. lijima, Phys. Rev. Lett., vol. 89, p. 155501-4, 2002.

78 B. Fiedler, F.H. Gojny, M.H.G. Wichmann, M.C.M. Nolte, K. Schulte, Composites Science and Technology, vol. 66, p. 3115-3125, 2006.

79 K.P. Ryan, M. Cadek, V. Nicolosi, D. Blond, M. Ruether, G. Armstrong, H. Swan, A. Fonseca, J.B. Nagy, W.K. Maser, W.J. Werner, J.N. Coleman, Composites science and Technology, vol. 67, p. 16401649, 2007. 
80 P. Pötschke, A.R. Bhattacharyya, A. Janke, European Polymer Journal, vol. 40, p. 137-148, 2004.

81 T. Uchida, S. Kumar, J. Appl. Polym. Sci., vol. 98, p. 985-989, 2005.

82 T.D. Fornes, J.W. Baur, Y. Sabba, E.L. Thomas, Polymer, vol. 47, p. 1704-1714, 2006.

83 J. Yu, K. Lu, E. Sourty, N. Grossiord, C.E. Koning, J. Loos, Carbon, vol. 45, p. 2897-2903, 2007.

84 K. Masenelli-Varlot, L. Chazeau, C. Gauthier, J.Y. Cavaillé, Composites Science and Technology, vol. 69, p. 1533-1539, 2009.

85 G.B. Thompson, M. Abdalla, D. Dean, Microscopy and Microanalysis, vol. 12, p. 1578-1579, 2006.

86 C. Sealy, Materials Today, vol. 9, issue 4, p. 10, 2006.

87 M. H. Gass, K. K. Koziol, A. H. Windle and P. A. Midgley, Nano Lett., vol. 6, p. 376-379, 2006.

88 Y. Liu, A. Bogner-Van De Moortèle, T. Epicier, K. Sato, T. Konno, p.313-314 in 'emc2012', vol. 2: Physical Sciences: Tools and Techniques, ed. D.J. Stokes et J.L. Hutchinson, RMS: London, (2012).

89 F. Dalmas, Ph-D thesis, Institut National Polytechnique de Grenoble, 2005, available online at : http://hal.archives-ouvertes.fr/tel-00012111/

90 F. Dalmas, R. Dendiével, L. Chazeau, J.Y. Cavaillé, C. Gauthier, Acta Materialia, vol. 54, p. 29232931, 2006.

91 O. Meincke, D. Kaempfer, H. Weickmann, C. Friedrich, M. Vathauer, H. Warth, Polymer, vol. 45, p. 739-748, 2004.

92 Y. Li, H. Shimizu, Macromolecules, vol. 42, p. 4287-4293, 2009.

93 B. Fragneaud, K. Masenelli-Varlot, A. González-Montiel, M. Terrones, J.Y. Cavaillé, Chemical Physics Letters, vol. 444, p. 1-8, 2007.

94 Z. F, S.G. Advani, Polymer, vol. 46, p. 5232-5240, 2005.

95 M. Dehonor Gomez, Ph-D thesis, Instituto Potosino de Investigacion Cientifica y Tecnologica (Mexico) and Institut National des Sciences Appliquées de Lyon (France), 2007. Available online at http://docinsa.insa-lyon.fr/these/pont.php?id=dehonor_gomez

96 C. Bower, R. Rosen, L. Jin, J. Han, O. Zhou, Appl. Phys. Lett. Vol. 74, p. 3317-3319, 1999.

97 D. Qian, E.C. Dickey, R. Andrews, T. Randell, Appl. Phys. Lett., vol. 76, p. 2868-2870, 2000.

98 O. Lourie, H.D. Wagner, Appl. Phys. Lett., vol. 73, p. 3527-3529, 1998.

99 F.H. Gojny, J. Nastalczyk, Z. Roslaniec, K. Schulte, Chem. Phys. Lett., vol. 370, p. 820-824, 2003.

100 G.L. Hwang, Y.T. Shieh, K.C. Hwang, Adv. Funct. Mater., vol. 14, p. 487-491, 2004.

101 C. Probst, R. Gauvin, R.A.L. Drew, Micron, vol. 38, p. 402-408, 2007.

102 M.R. Maschmann, Q. Zhang, R. Wheeler, F. Du, L. Dai, J. Baur, Appl. Mater. Interfaces, vol. 3, p. 648-653, 2011.

103 W. K. Wong, A. Nojeh, R.F.W. Pease, Scanning, vol. 28, p. 219-227, 2006.

104 T. Brintlinger, Y.F. Chen, T. Durkop, E. Cobas, M. S. Fuhrera, J. D. Barry, J. Melngailis, Appl. Phys. Lett., vol. 81, p. 2454-2456, 2002.

105 Y. Homma, S. Suzuki, Y. Kobayashi, M. Nagase, D.Takagi, Appl. Phys. Lett., vol. 84, p. 1750-1752, 2004.

106 F. Dalmas, L. Chazeau, C. Gauthier, K. Masenelli-Varlot, R. Dendievel, J.-Y. Cavaillé, L. Forró, Journal of Polymer Science: Part B: Polymer Physics, vol. 43, p. 1186-1197, 2005.

107 F. Dalmas, J.-Y. Cavaillé, C. Gauthier, L. Chazeau, R. Dendievel, Compos. Sci. Technol., vol. 67, p. 829-839, 2007.

108 B. Fragneaud, K. Masenelli-Varlot, A. González-Montiel, M. Terrones, J.-Y. Cavaillé, Compos. Sci. Technol., vol. 68, p. 3265-3271, 2008.

109 W. Chen, X. Tao, Appl. Surf. Sci., vol. 252, p. 3547-3552, 2006. 
110 J. Loos, A. Alexeev, N. Grossiord, C. E. Koning, O. Regev, Ultramicroscopy, vol. 104, p. 160-167, 2005.

111 P. Gentsch, H. Gilde, L. Reimer, Journal of Microscopy, vol. 100, p. 81-92, 1974.

112 A. Takaoka, T. Hasegawa, International Microscopy Congress 16 Sapporo, Japan (2006)

113 A. Bogner, P.-H. Jouneau, G. Thollet, D. Basset, C. Gauthier, Micron, vol. 38, p. 390-401, 2007.

114 A. Bogner, G. Thollet, D. Basset, P.H. Jouneau, C. Gauthier, Ultramicroscopy, vol. 104, p. 290-301, 2005.

115 P. Jornsanoh, G. Thollet, K. Masenelli-Varlot, C.Gauthier, FR Patent 06-09-708, 2006.

116 P. Jornsanoh, G. Thollet, J. Ferreira, K. Masenelli-Varlot, C. Gauthier, A. Bogner, Ultramicroscopy, vol. 111, p. 1247-1254, 2011.

117 K. Masenelli-Varlot, A. Malchère, J. Ferreira, H. Heidari Mezerji, S. Bals, C. Messaoudi, S. Marco, Microsc. Microanal., in press.

118 Y. Liu, PhD thesis, INSA-Lyon, 2013 (to be soon available on http://theses-search.insa-lyon.fr/).

119 B. J. Inkson, M. Mulvihill, G. Möbus, Scripta Materialia, vol. 45, p. 753-758, 2001.

120 L. Holzer, M. Cantoni, p. 410-435 in 'Nanofabrication using focused ion and electron beams: Principles and applications', eds. Utke I., Monshkalev S.A., Russell P., Oxford University Press, New York, 2012.

121 A.J. Kubis, G.J. Shiflet, D.N. Dunn, R. Hull, Metallurgical and Materials Transactions A, vol. 35 A, p. 1935-1943, 2004.

122 V. Salles, T. Fiorido, J. Galineau, L. Seveyrat, F. Belhora, P.J. Cotitnet, L. Hu, Y. Liu, B. Guiffard, A. Bogner-Van De Moortele, T. Epicier, D. Guyomar, A. Brioude, to appear in Sensors and Actuators A., 2014.

123 R.S. Rajeev, E. Harkin-Jones, K. Soon, T. McNally, G. Menary, C.G. Armstrong, P.J. Martin, Materials Letters, vol. 62, p. 4118-4120, 2008.

124 D. J. Stokes, F. Morrissey, B. H. Lich, Journal of Physics: Conference Series, vol. 26, p. 50-53, 2006. 125 J. Fu, S.B. Joshi, J.M. Catchmark, J. of Micromechanics and Microengineering, 18, 1-8, 2008.

\subsection{Figures}

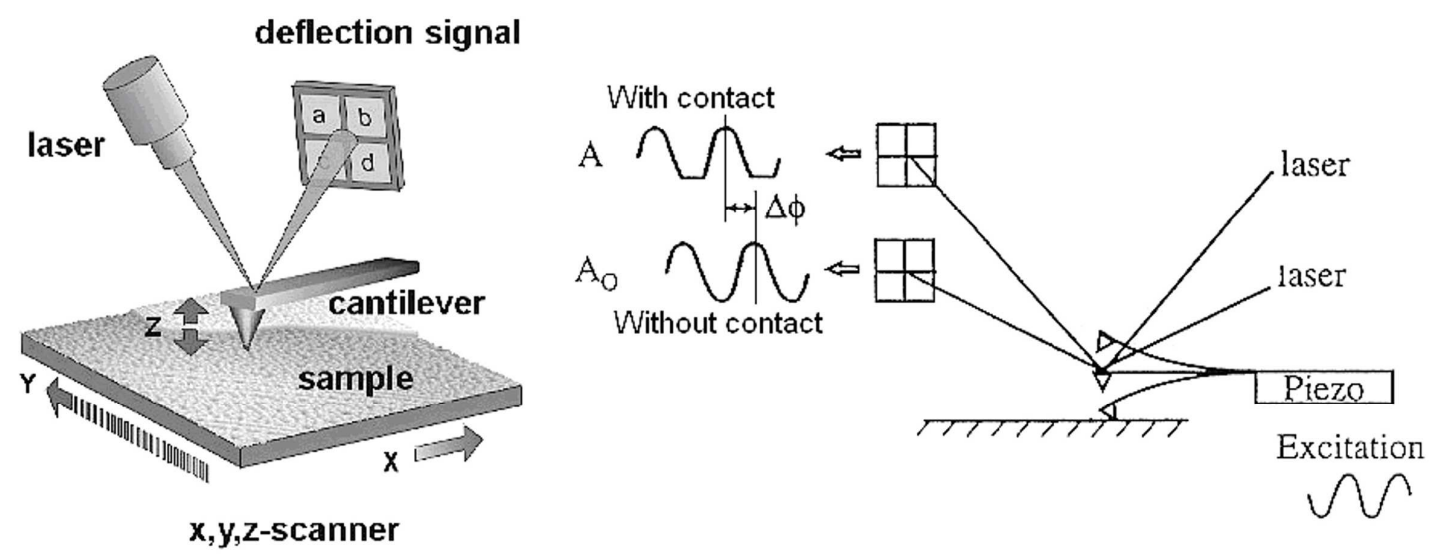

Figure 13.1: schematic presentation of AFM device. 

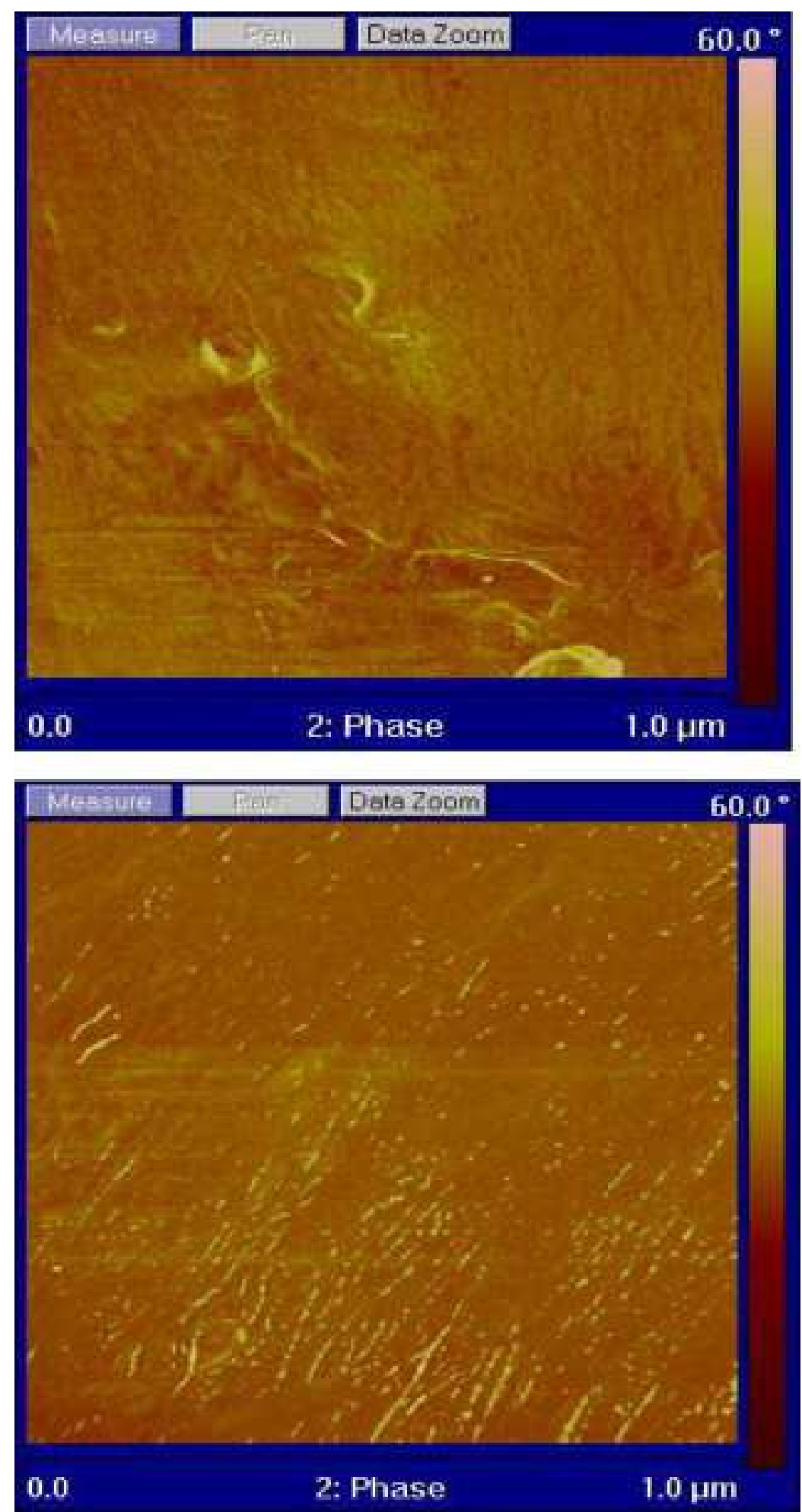

Figure 13.2: AFM image of a a) a PE and b) PE with $10 \mathrm{wt} \%$ MWCNT. The samples were taken parallel to the extrusion flow direction, with permission from Elsevier [24]. 


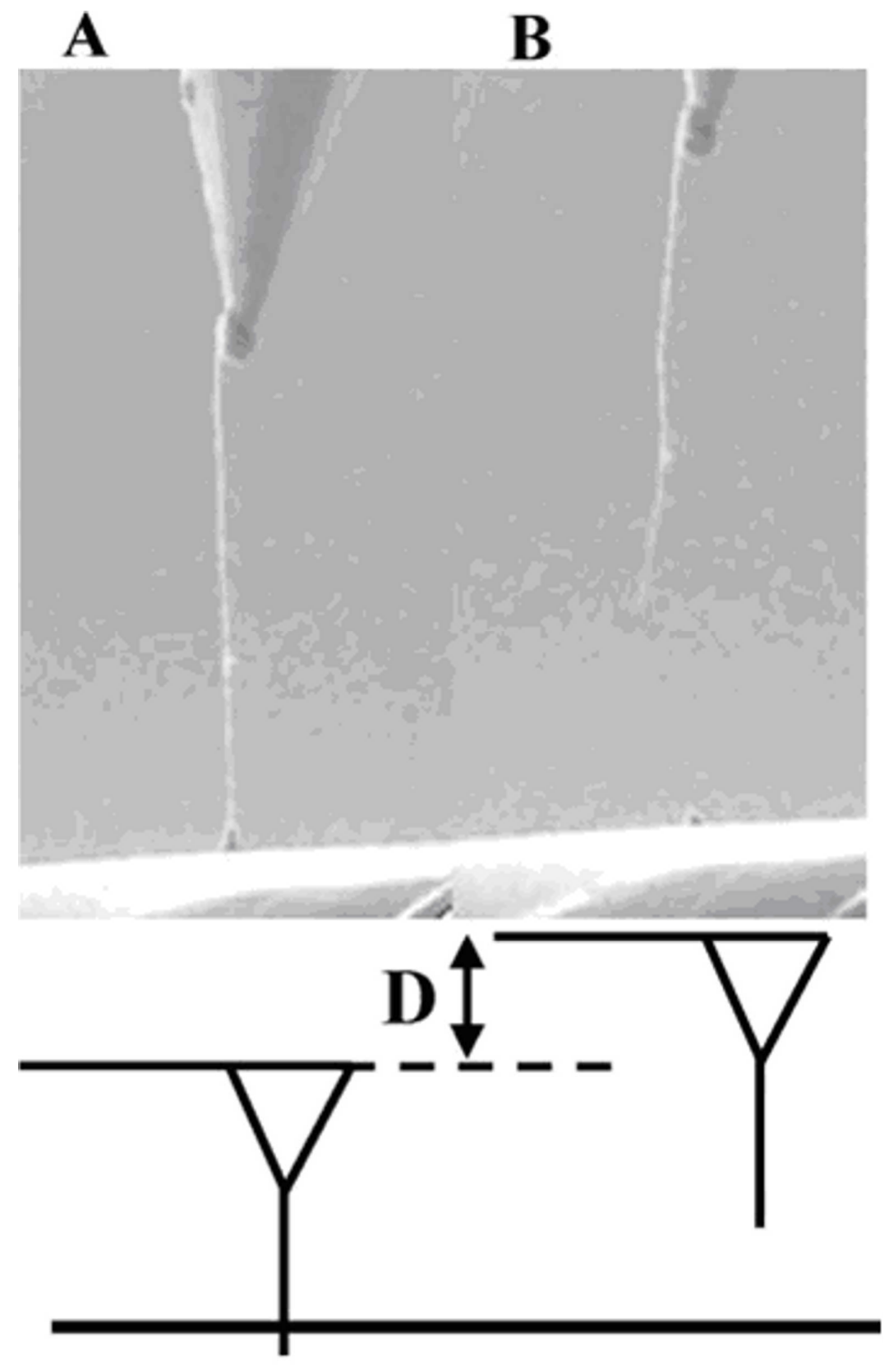

\section{POLYMER}

Figure 13.3: SEM images of an AFM cantilever used to calculate nanotube pullout forces. A) The nanotube is partially embedded within the solid polymer matrix. The AFM chip, containing the AFM cantilever and tip, is then translated away from the polymer surface, resulting in continued bending of the cantilever. The nanotube pulls out of the polymer at a critical bending of the cantilever (B). This bending deflection, $\mathrm{D}$, is calculated from the position of the cantilever before and after the pullout. The nanotube embedded length is calculated by the difference between the nanotube free length in (A) and (B). With permission from Wiley and sons [31] 


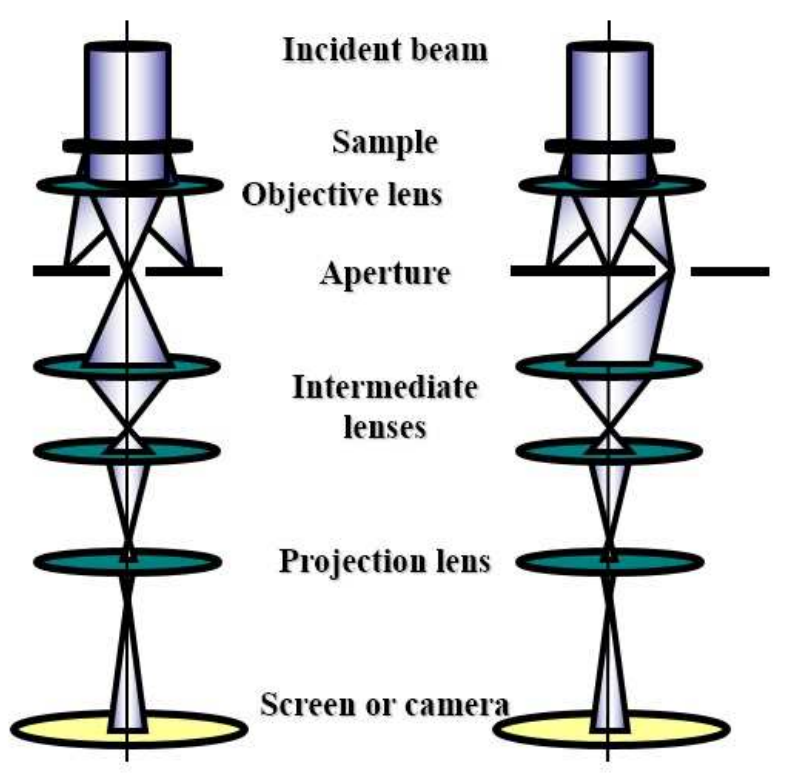

a)

b)

Figure 13.4: schematic presentation of the TEM a) bright field and b) dark field modes. The difference lies in the position of the aperture: in the bright field mode, the central spot of the diffraction pattern is selected whereas the dark field mode is based on the selection of a diffraction spot.
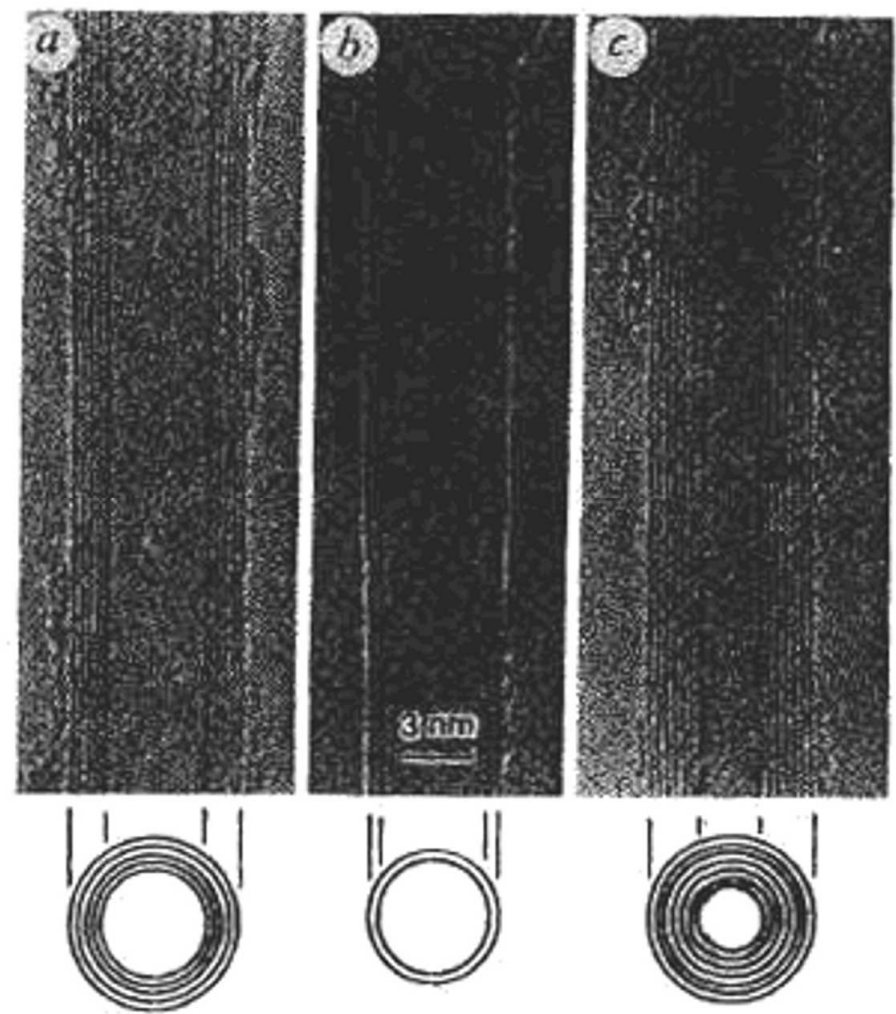

Figure 13.5: first TEM pictures of carbon nanotubes [2], with permission from Nature Publishing Group. 


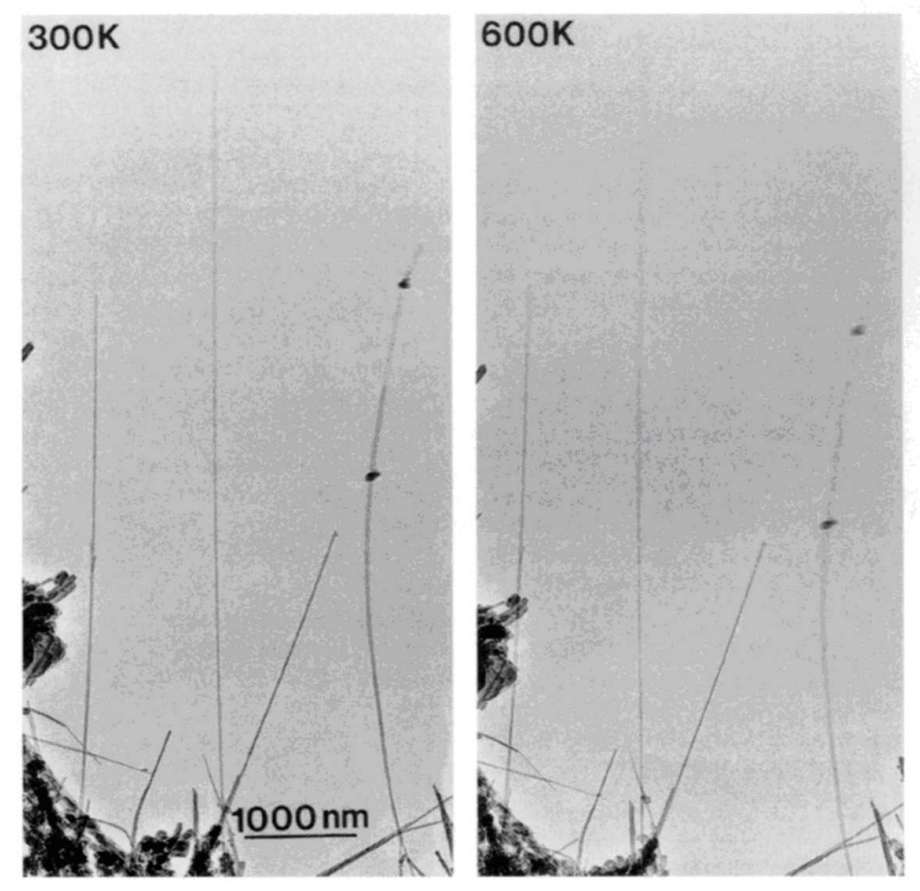

Figure 13. 6: TEM micrographs showing the vibration of carbon nanotubes like clamped cantilevers, from [71], with permission from Nature Publishing Group. 

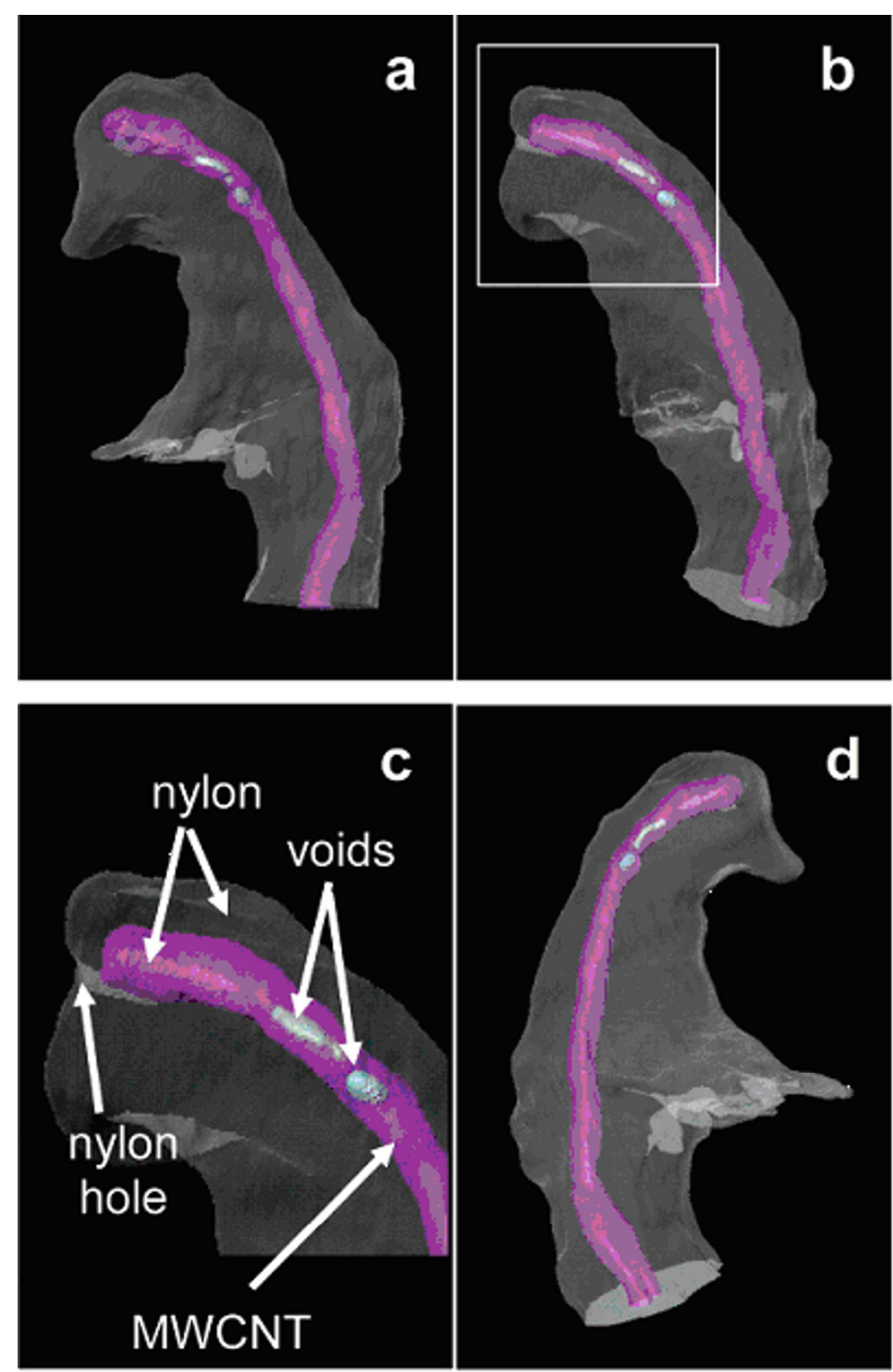

Figure 13.7: carbon nanotube embedded in a nylon matrix, observed by electron tomography [87], with permission from the Americal Chemical Society.
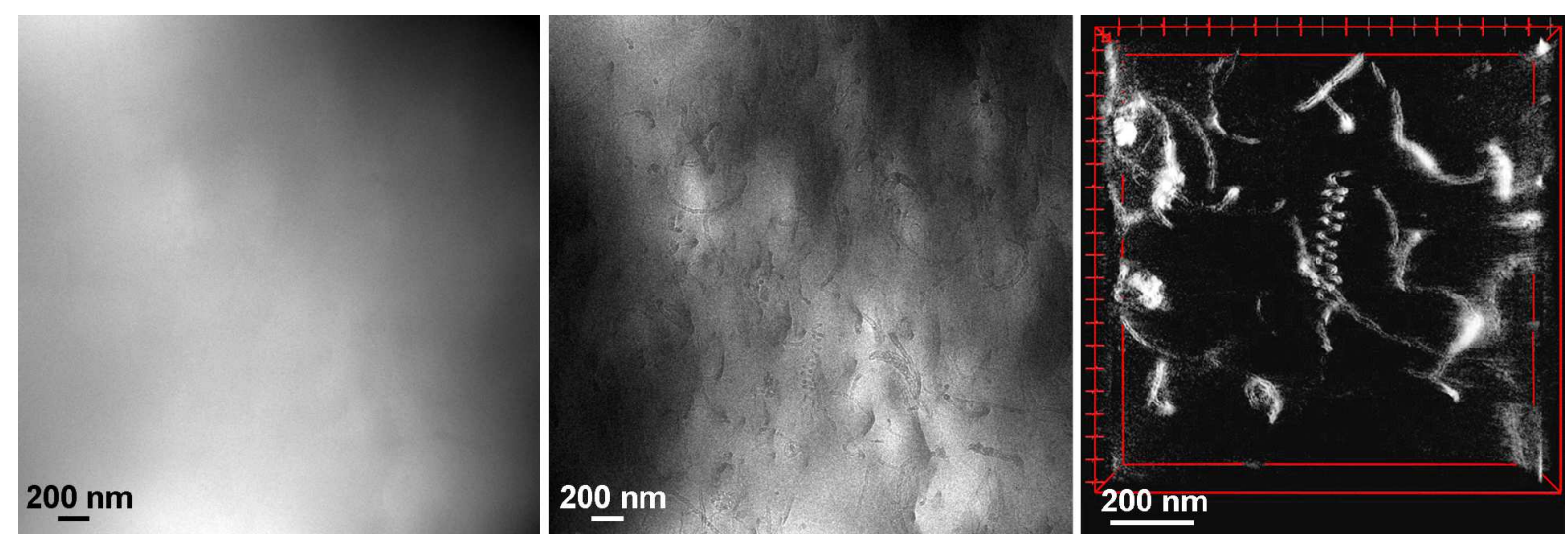

Figure 13.8: EFTEM tomography of a P(S-BuA) - 3\% CNTs latex nanocomposite ( $\mathrm{C}_{\mathrm{s}}$-corrected Titan-FEI operated at $80 \mathrm{kV}$ ). The micrographs image the same area of a $200 \mathrm{~nm}$ thick ultramicrotomic section inclined at $70^{\circ}$ with a defocus of $-5 \mu \mathrm{m}$, without and with zero-loss filtering. A 3D rendering of a representative reconstructed volume is shown on the right (reverse contrast for a better visibility). 


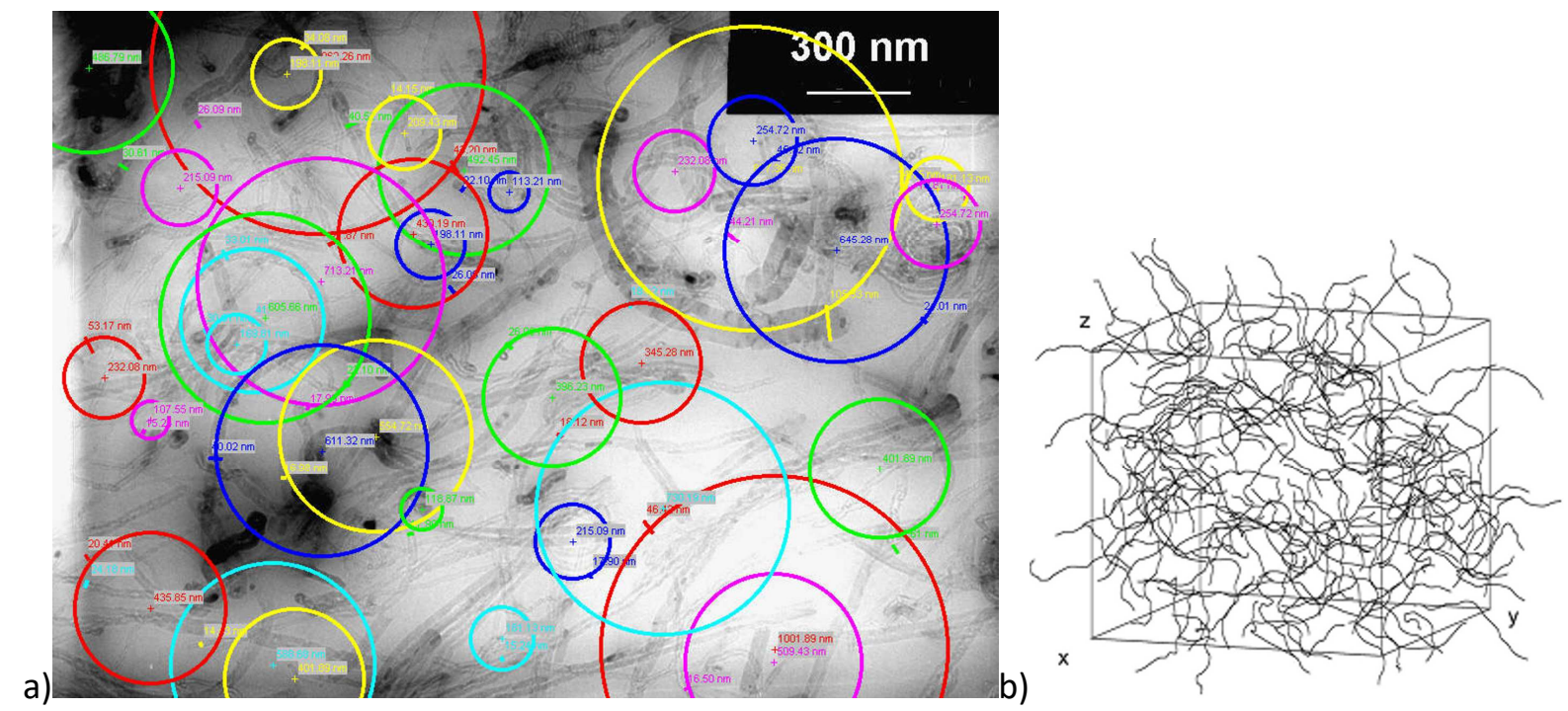

Figure 13. 9: a) measurement of the 2D apparent distribution of the nanotube curvature radius in polymer/nanotube composites [89]. b) example of 3D nanotube network [90].

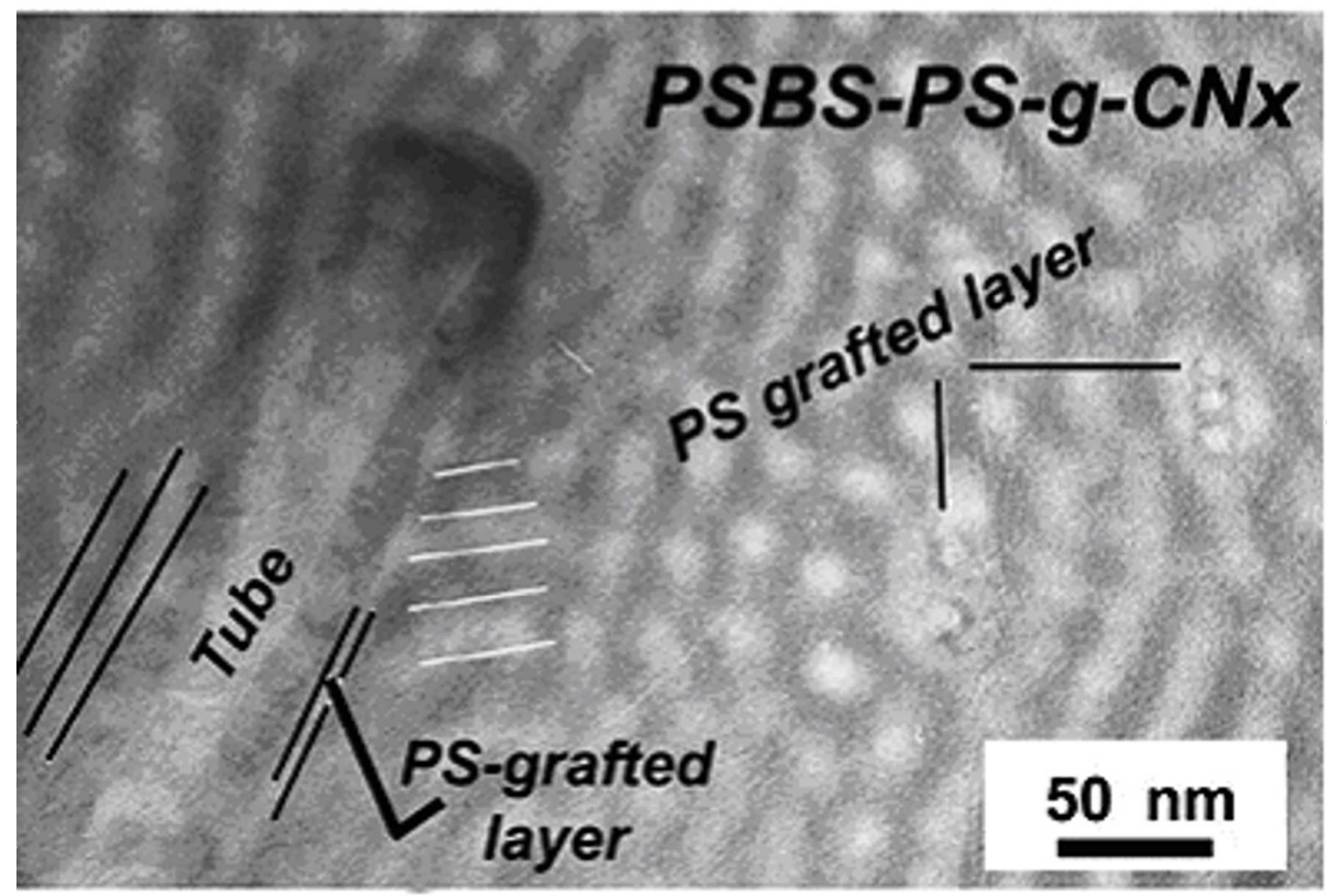

Figure 13.10: TEM bright-field image of a SBS copolymer filled with 2.5 wt.\% of polystyrene-grafted nitrogen-doped carbon nanotubes after staining with $\mathrm{RuO}_{4}$. 


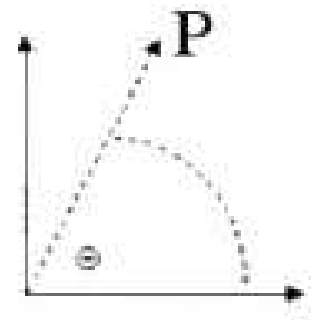

$a_{i j}=\left\langle P_{i} P_{j}\right\rangle$

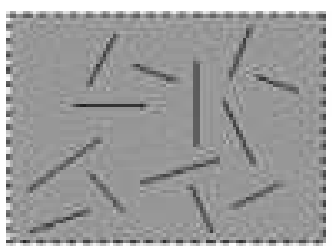

$a_{i f}=\left[\begin{array}{cc}0.5 & 0 \\ 0 & 0.5\end{array}\right]$

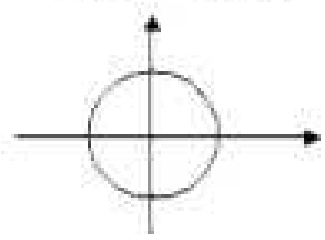

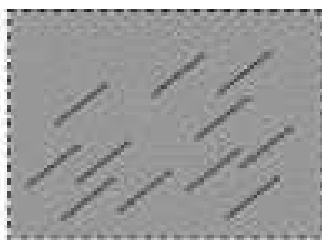

$a_{i j}=\left[\begin{array}{ll}0.5 & 0.5 \\ 0.5 & 0.5\end{array}\right]$

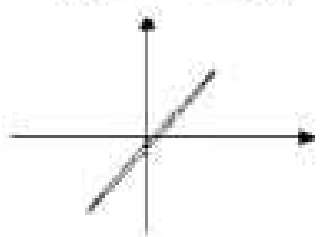

Figure 13.11: Characterization of the nanotube orientation state in one TEM image by a second order tensor and an ellipse, with permission from Elsevier [94].

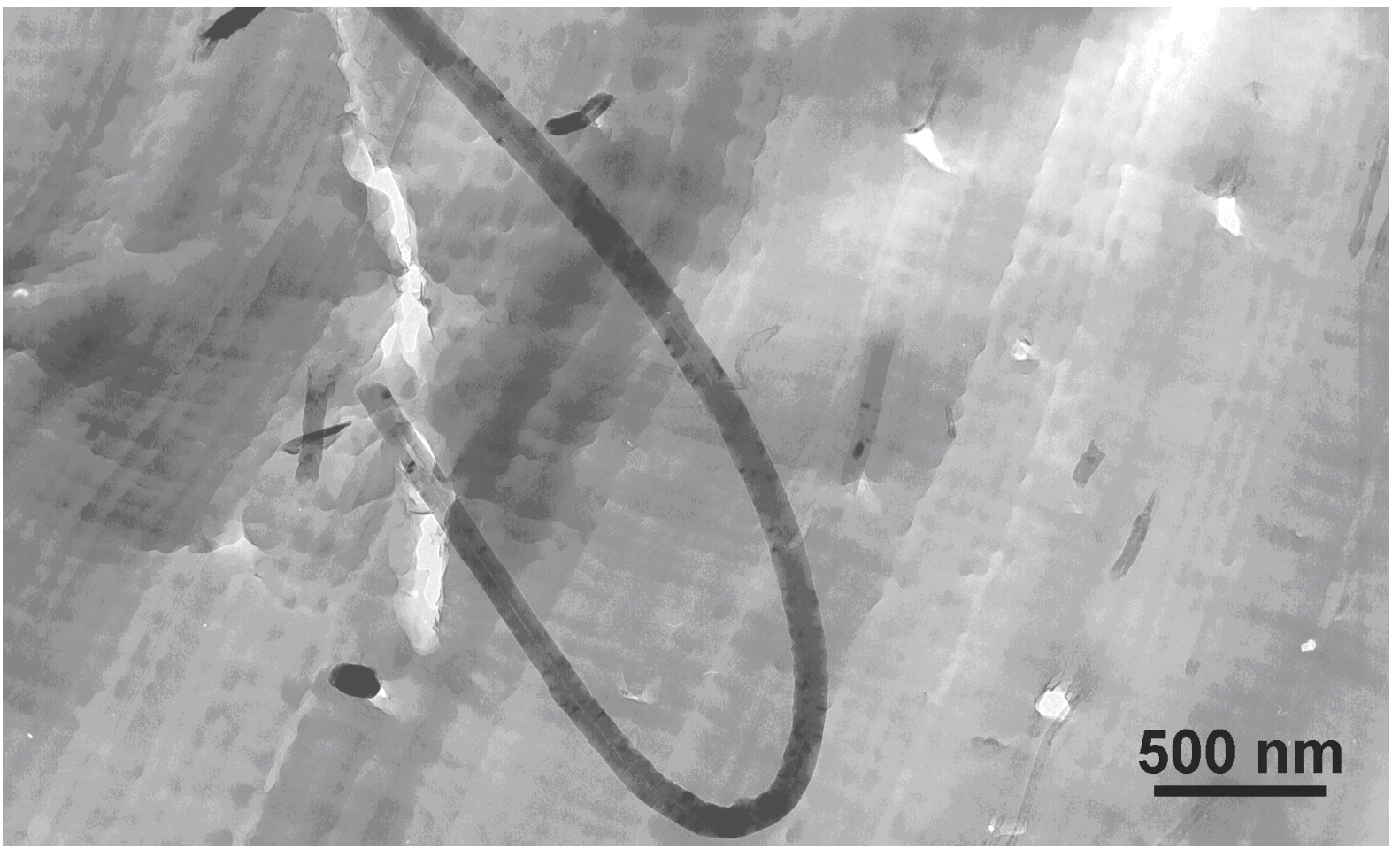

Figure 13.12: TEM bright-field images of composites made of polystyrene and a) raw and b) polystyrene-grafted nitrogen-doped multiwalled nanotubes [95]. 


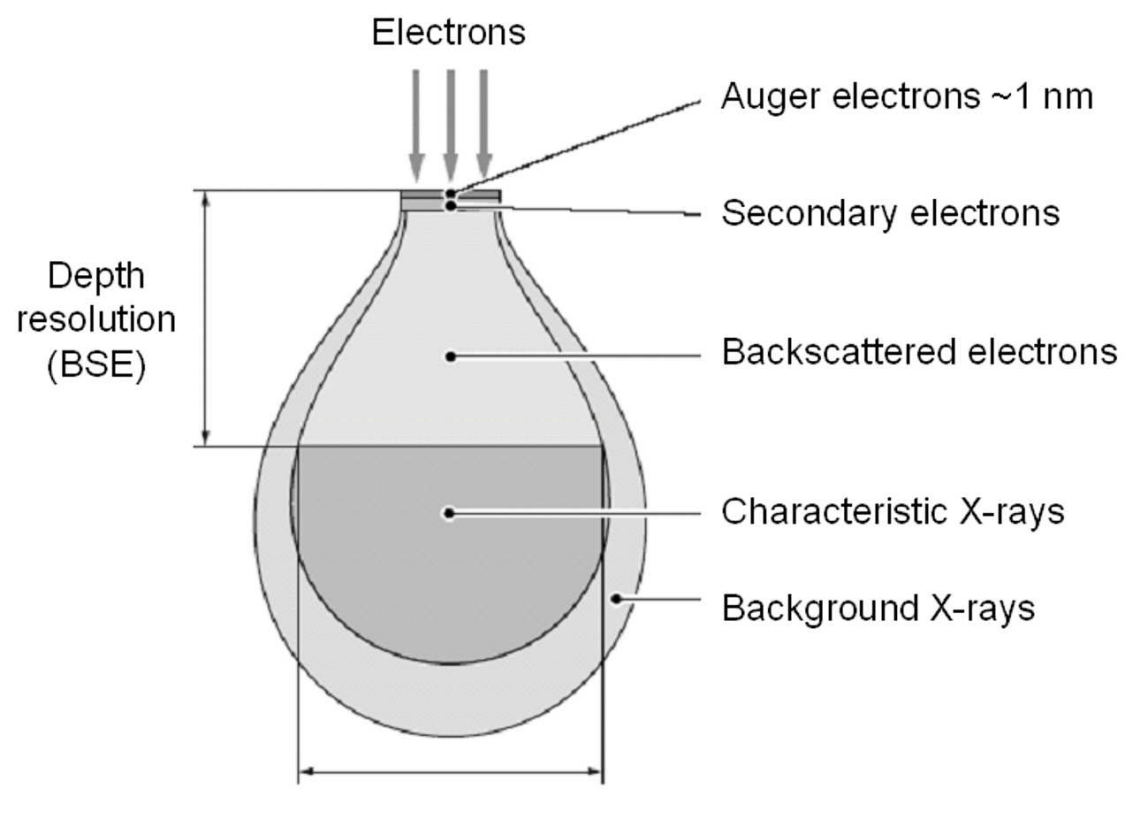

Lateral resolution (BSE)

Figure 13.13: Signals detected in SEM, resulting from electrons/sample interaction.

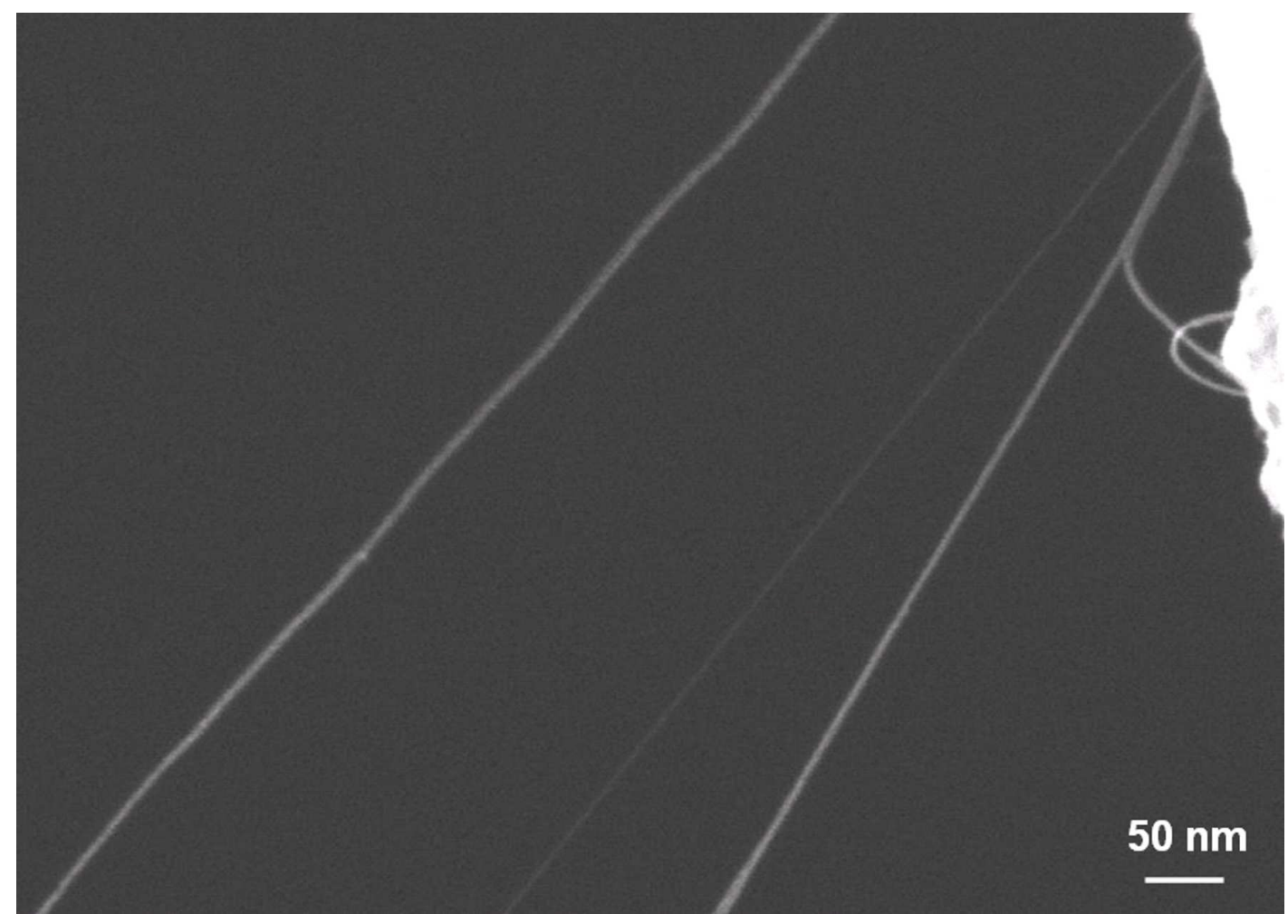

Figure 13.14: SEM image of individual SW and small bundles of SW acquired in STEM mode. 

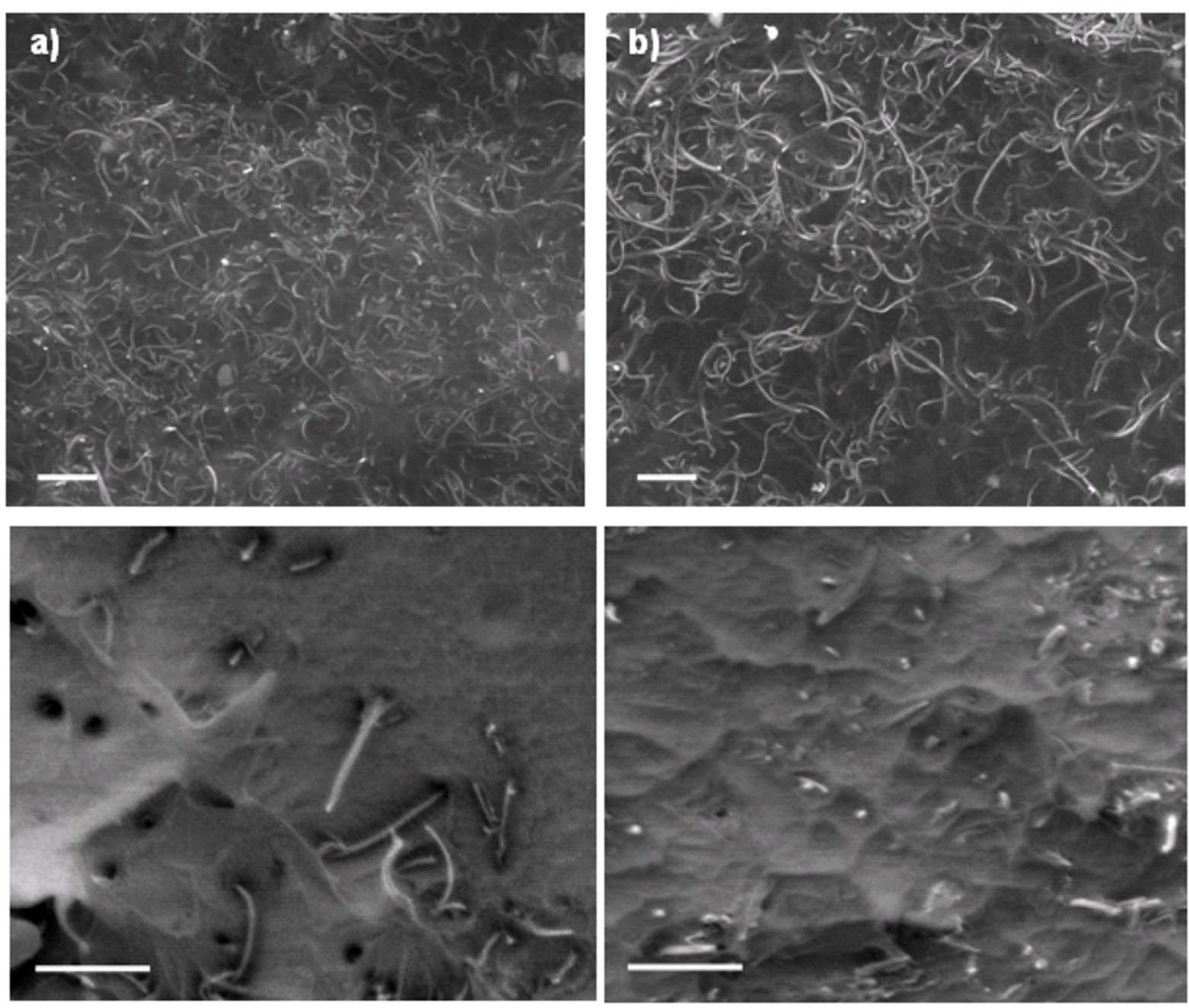

Figure 13.15: CNT/polymer nanocomposites observed in SEM : a) and b) $\mathrm{P}(\mathrm{S}-\mathrm{ABu}) / \mathrm{MW}$ CNT films surface respectively prepared by evaporation and film formation or freeze-drying and hot-pressing but showing similar fillers distribution; c) and d) PS matrix containing ungrafted or PS-grafted Ndoped CNT; a fracture performed at ambient temperature highlights the difference in fillers/matrix interface strength. Scale bars: $1 \mu \mathrm{m}$. 

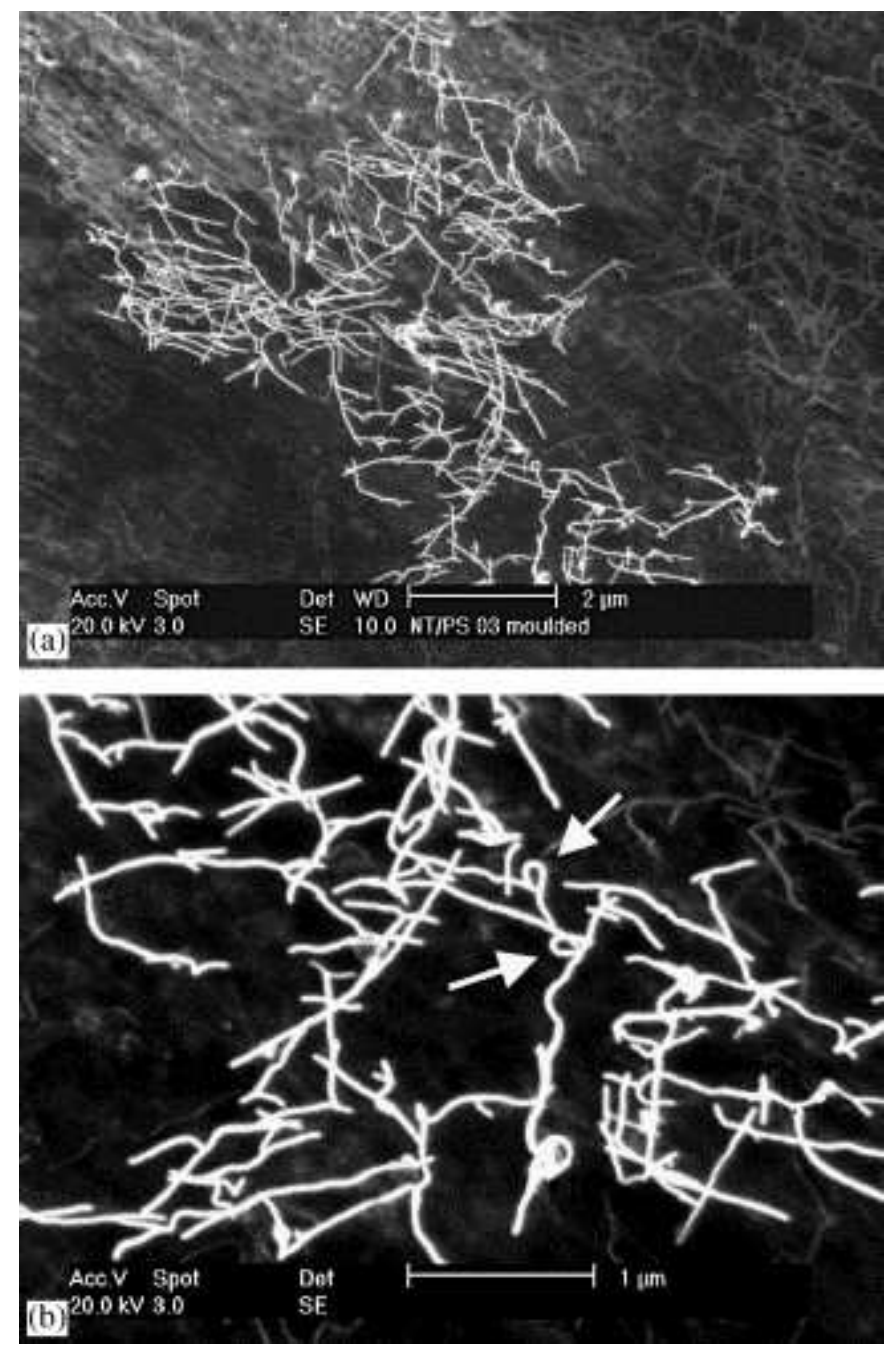

Figure 13.16: Charge-Contrast Imaging in a nanocomposite film of PS containing $0.3 \mathrm{wt} \%$ of SW CNT. with permission from Elsevier [110]

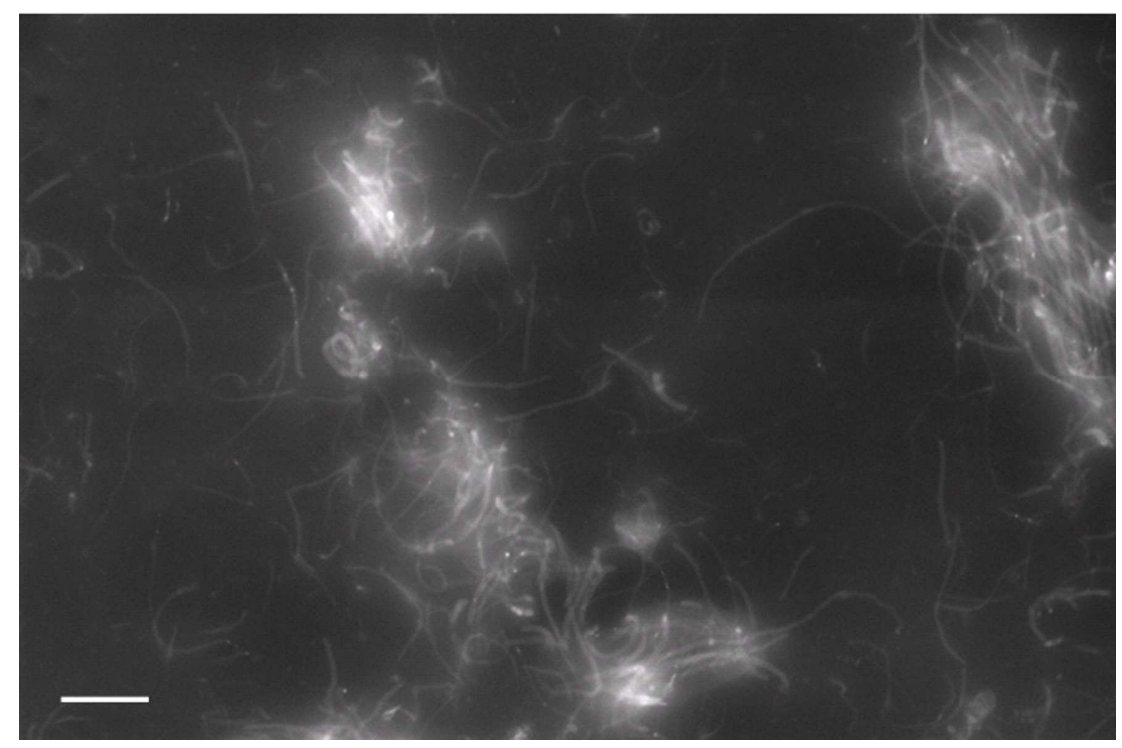

Figure 13.17: STEM image performed in SEM on cryo-ultramicrotomic sections of $P(S-A B u) / M W C N T$ nanocomposites films in annular dark-field conditions at $30 \mathrm{kV}$ : the contrast between the fillers and the matrix is important. Scale bar: $500 \mathrm{~nm}$. 


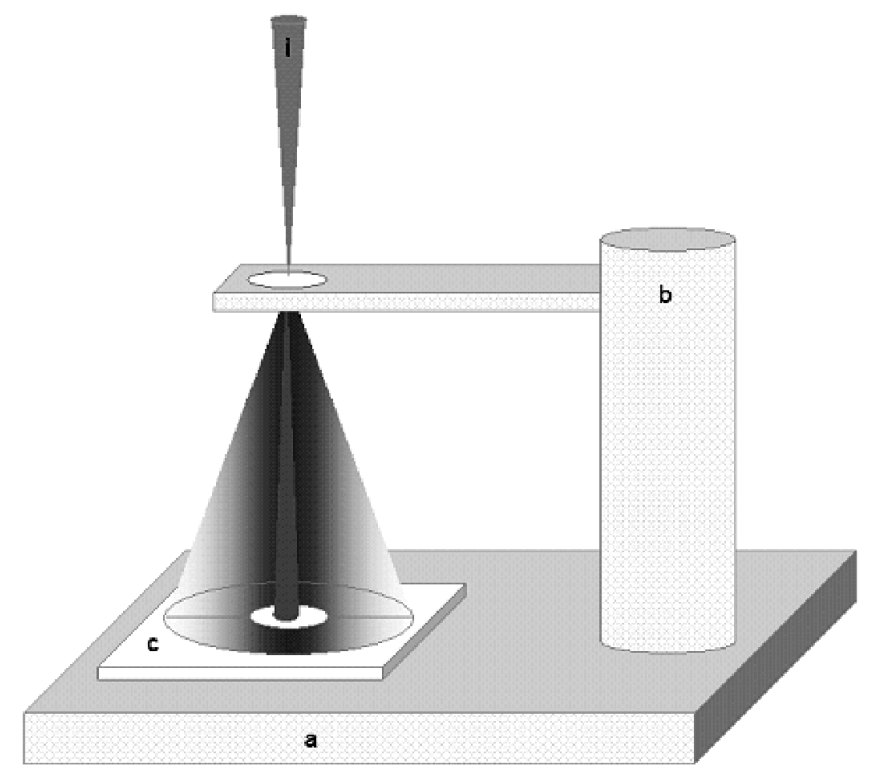

Figure 13.18: Scheme of the wet-STEM imaging mode. a: Peltier cooling stage; b: TEM-grid type sample holder; c: annular detector for transmitted electrons collection; i: incident electron beam.

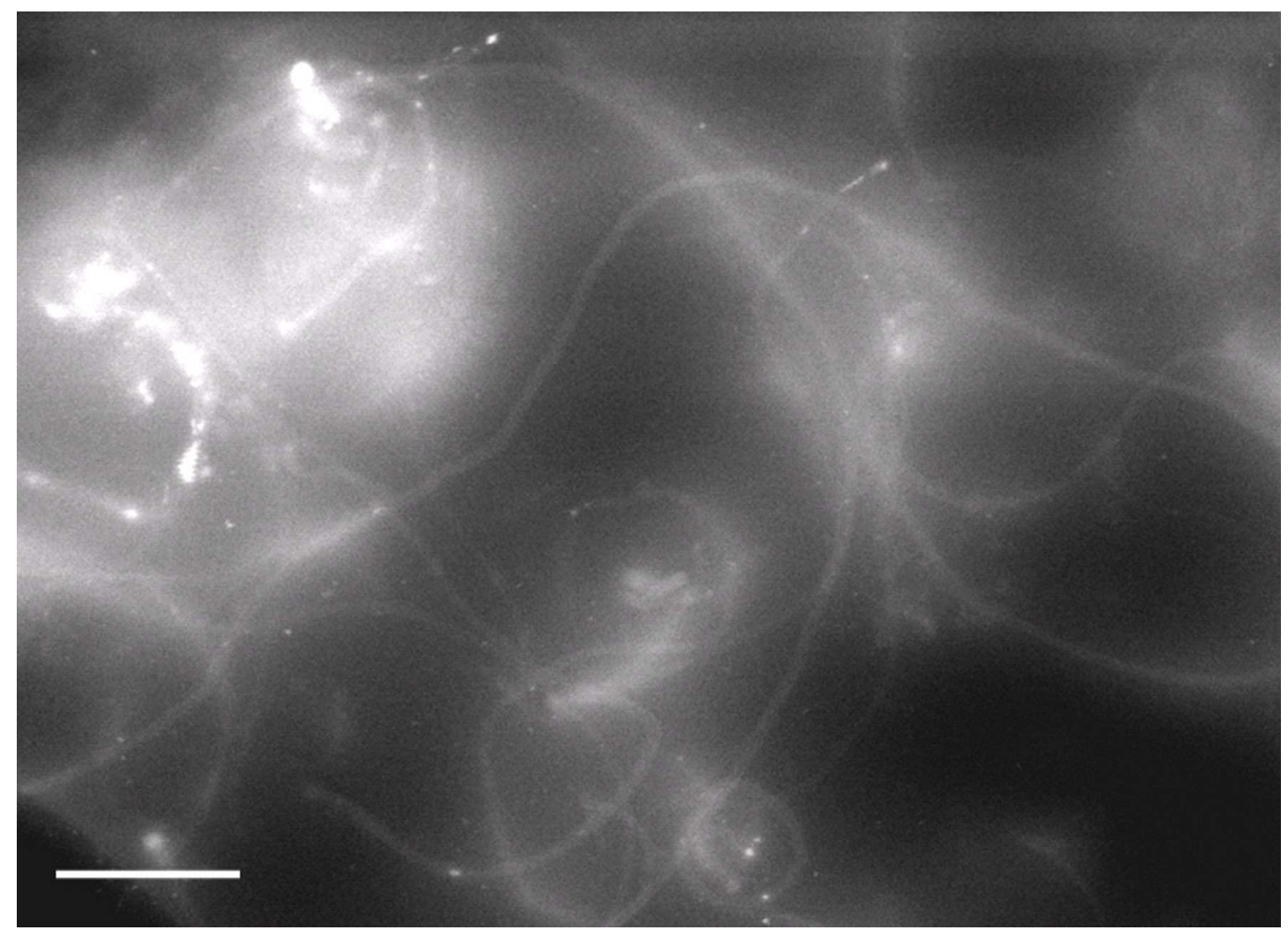

Figure 13.19: Wet-STEM image of MW nanotubes and surfactant dispersed in water. 


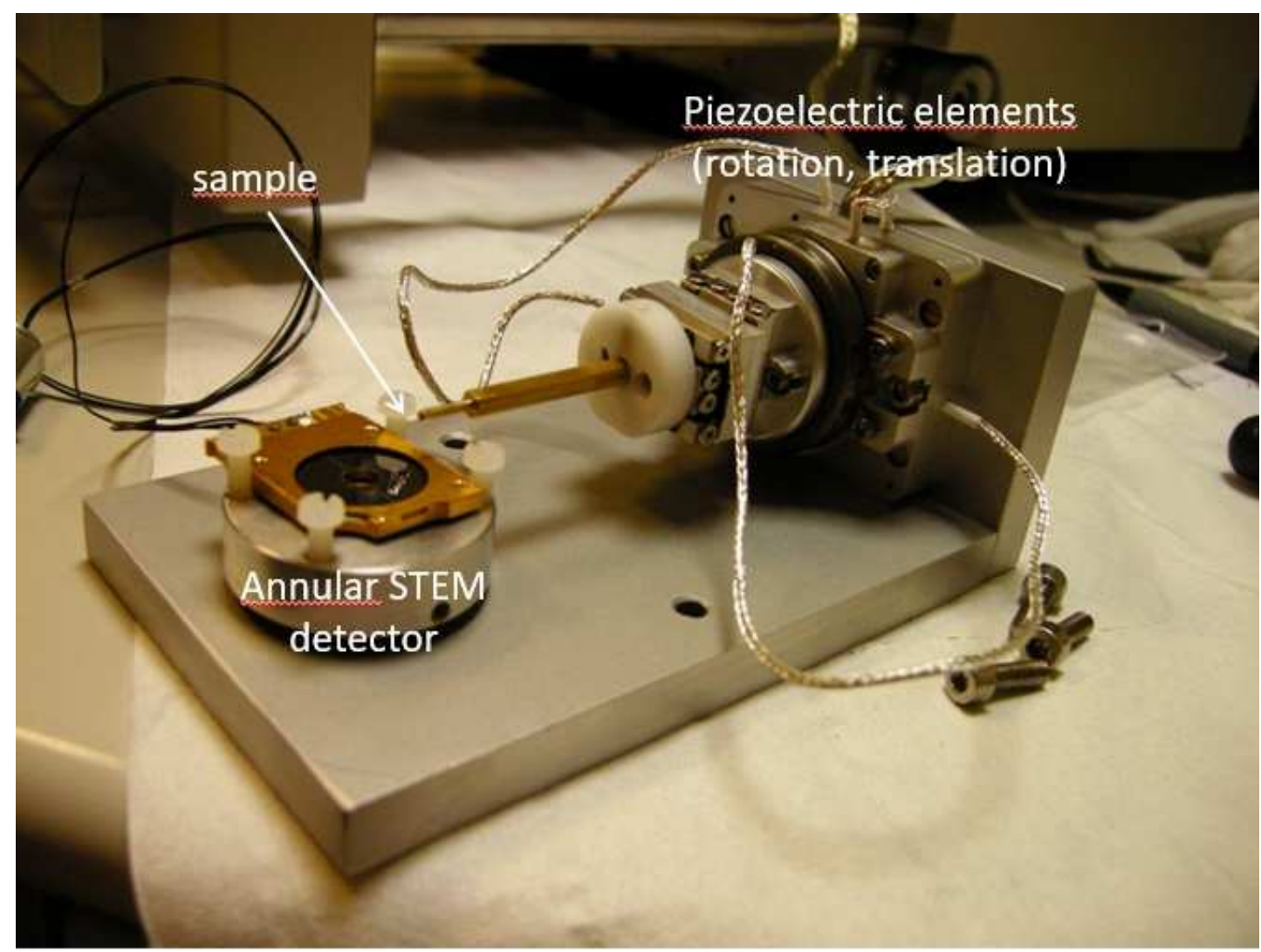

Figure 13.20: Principles of electron tomography in a ESEM through STEM observations at different tilt angles.
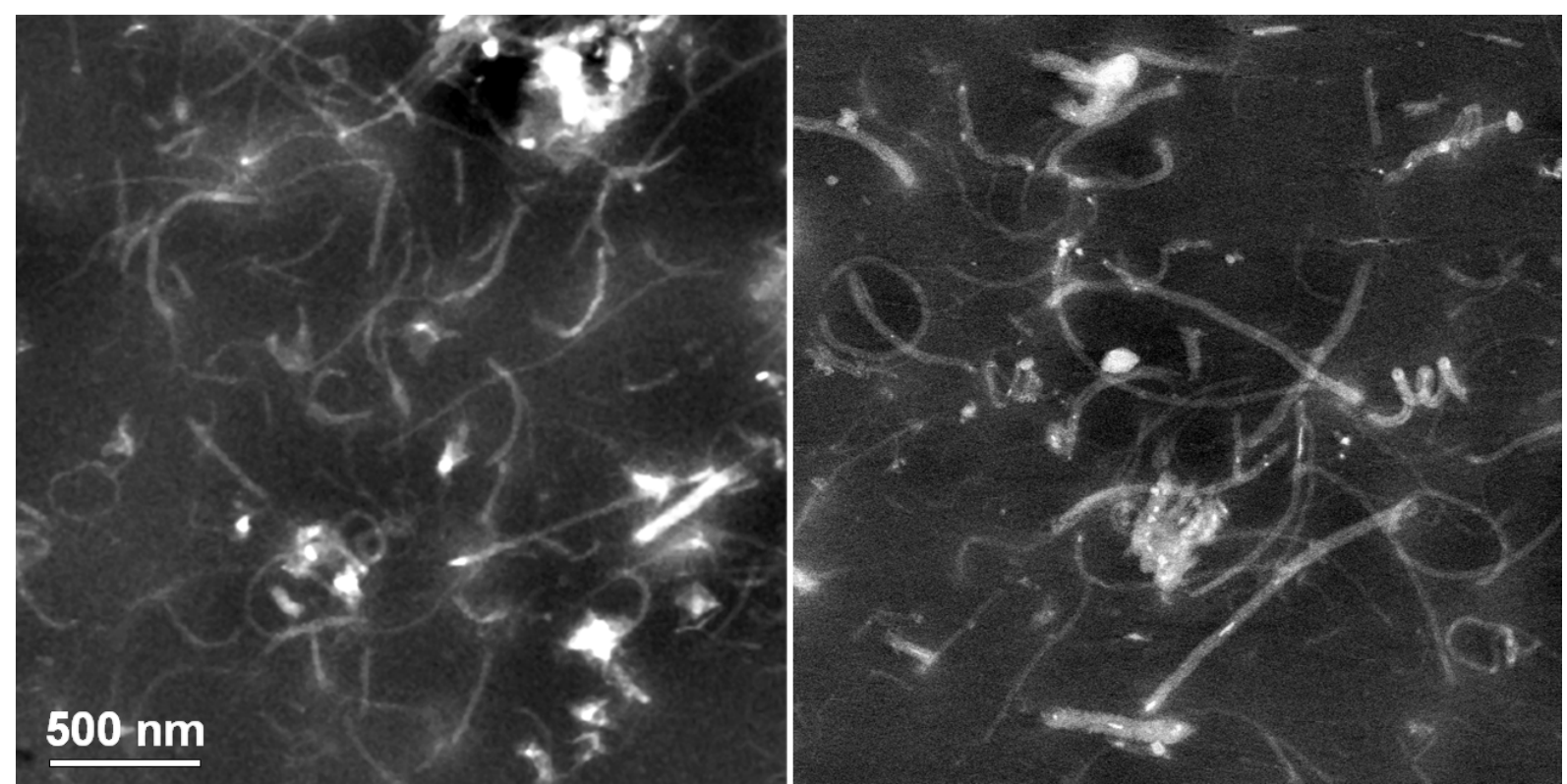

Figure 13.21: low-voltage STEM images of P(S-BUA) - 3\% CNTs latex nanocomposites. Left: ESEM (FEI XL30 image), right: HR-SEM Hitachi S5500. 

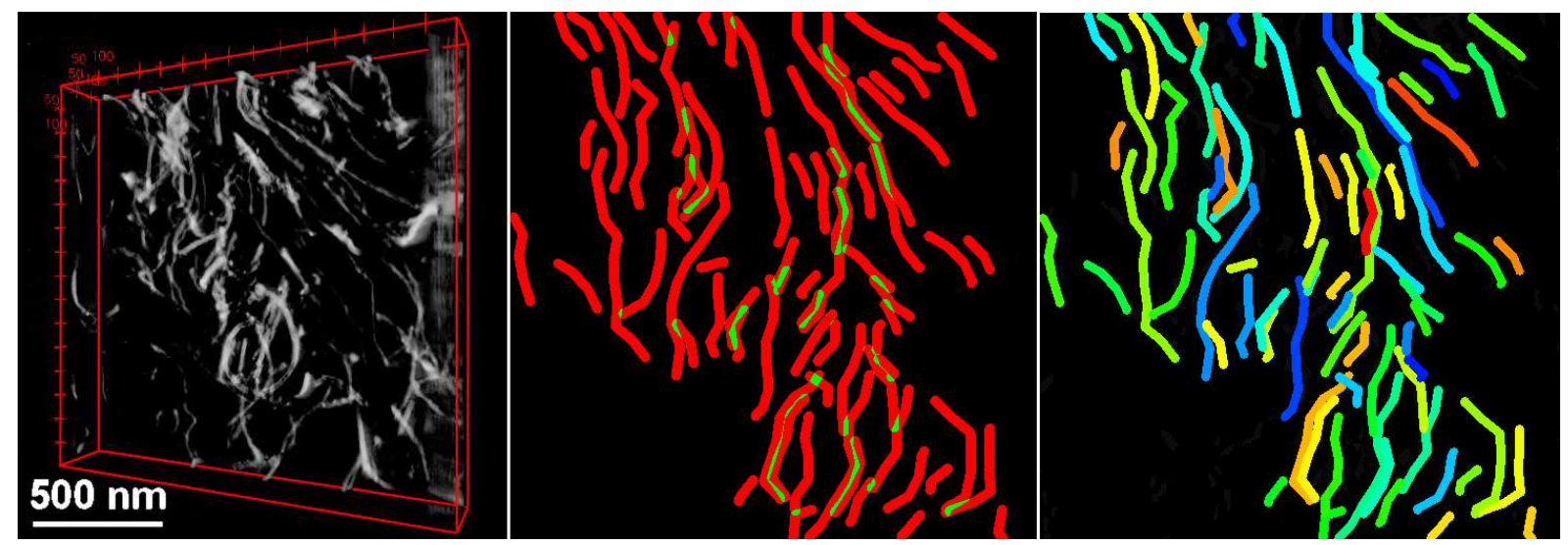

Figure 13.22: 3D rendering of a tomographic reconstruction from a series of SEM images recorded in the transmission between $+40^{\circ}$ and $-40^{\circ}$ (maximum tilting range permitted in a Hitachi S5500 high resolution microscope). Diagrams on the right refer to a $2 \mathrm{~S}$ slice of the tomogram: they identify individual nanotubes (middle) and contact areas (right; see text for details).
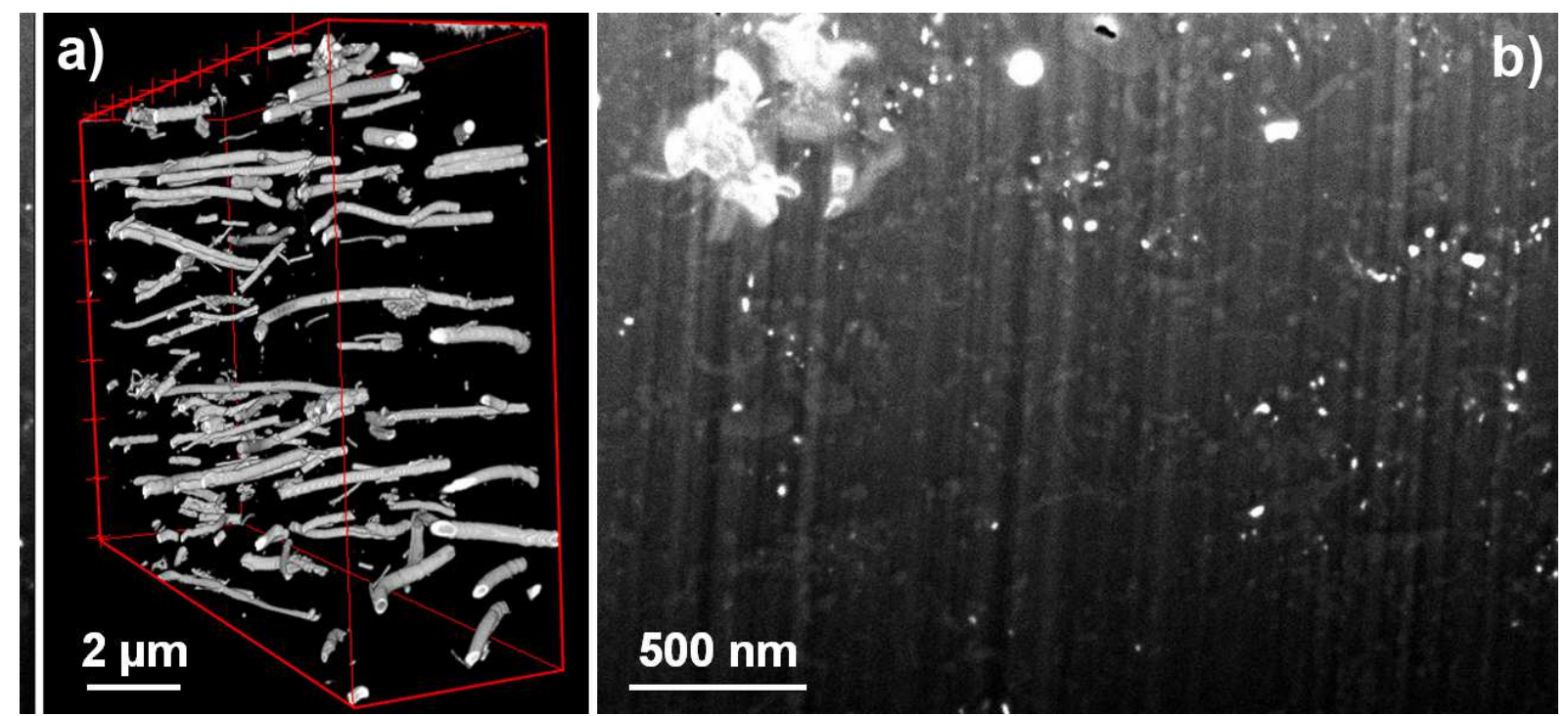

Figure 13.23: FIB imaging (Zeiss NVision40) of polymer-based nanocomposites; a): 3D reconstruction of a volume (about $20 \times 14 \times 7 \mu^{3}$ - the shorter length corresponds to the slicing direction -) from a polyurethane (PU) matrix containing (2.5 wt\%) $\mathrm{Fe}_{3} \mathrm{C}$ carbide-based nanowires fabricated by electrospinning [122]; b): low voltage (2 kV) SEM image of a surface freshly FIB-milled at low current during an attempt to perform a 3D FIB-SEM volume characterization of a polymer-CNTs nanocomposite as shown in Figure 13.. 